\title{
COMPLETE ANALYSIS OF A DISCRETE-TIME BATCH SERVICE QUEUE WITH BATCH-SIZE-DEPENDENT SERVICE TIME UNDER CORRELATED ARRIVAL PROCESS: D-MAP $/ G_{n}^{(a, b)} / 1$
}

\author{
Umesh Chandra Gupta ${ }^{1, *}$, Nitin Kumar ${ }^{1}$, Sourav Pradhan ${ }^{2}$, \\ Farida Parvez Barbhuiya ${ }^{3}$ and Mohan L. Chaudhry ${ }^{4}$
}

\begin{abstract}
Discrete-time queueing models find a large number of applications as they are used in modeling queueing systems arising in digital platforms like telecommunication systems and computer networks. In this paper, we analyze an infinite-buffer queueing model with discrete Markovian arrival process. The units on arrival are served in batches by a single server according to the general bulkservice rule, and the service time follows general distribution with service rate depending on the size of the batch being served. We mathematically formulate the model using the supplementary variable technique and obtain the vector generating function at the departure epoch. The generating function is in turn used to extract the joint distribution of queue and server content in terms of the roots of the characteristic equation. Further, we develop the relationship between the distribution at the departure epoch and the distribution at arbitrary, pre-arrival and outside observer's epochs, where the first is used to obtain the latter ones. We evaluate some essential performance measures of the system and also discuss the computing process extensively which is demonstrated by some numerical examples.
\end{abstract}

Mathematics Subject Classification. 60K25, 68M20.

Received August 19, 2020. Accepted April 5, 2021.

\section{INTRODUCTION}

Queueing models involving batch service have been investigated by many researchers in the past due to their potential applications in several stochastic systems. Chaudhry and Templeton [12] and Medhi [20] provide a detailed discussion on different types of bulk queueing models. The general bulk service rule finds applications in the field of manufacturing and production systems, where the server starts service with a batch of minimum threshold size " $a$ " and a maximum size " $b$ ". Moreover, the instances when the service rate (or service time) is dependent on the size of the batch being served are more appropriate in modeling many of the real world problems. Such queues are known as batch-size dependent service queues and play a vital role in group screening

Keywords. Batch-size dependent, discrete-Markovian arrival process, discrete-time, general bulk service, phase-type distribution.

1 Department of Mathematics, Indian Institute of Technology Kharagpur, Kharagpur 721302, India.

2 Department of Mathematics, Visvesvaraya National Institute of Technology Nagpur, Nagpur 440010, India.

3 Department of Mathematics, Birla Institute of Technology and Science, Pilani, Hyderabad Campus, Hyderabad 500078, India.

4 Department of Mathematics and Computer Science, Royal Military College of Canada, P.O. Box 17000, STN Forces, Kingston, ON K7K7B4, Canada.

* Corresponding author: umesh@maths.iitkgp.ac.in 
of blood or urine samples for a particular disease, say HIV (see Abolnikov and Dukhovny [1] as well as Bar-lev et al. $[5,6])$. A group, if found infected by the disease, is set for further testing which may occur individually. If the size of the batch is large, then testing blood sample may take longer time which is a direct application of batch-size dependent service. Moreover, in modern telecommunication systems, the transfer of information (data, voice, videos, and images) occurs in batches of packets where the transmission time depends on the batch size of packets. In recent years, many researchers have focussed on studying batch-size dependent service queues, both with finite buffer (see Yu and Alfa [27] as well as Banerjee et al. [4]) and infinite buffer (see Claeys et al. $[14,15]$ as well as Pradhan and Gupta [21,22]). Claeys et al. [15] provided the application of batch-size dependent service policy mainly in the area of telecommunication system and illustrated the effect of neglecting batch-size dependent service times on the performance measures of the system.

In many real-world queueing systems, the arrival of customers or units does not occur independent of each other. As for instance, in telecommunication systems the transmission of information, in the form of packetized data, takes place with a very high speed over a large network which exhibits burstiness, correlation and selfsimilarity. These features cannot be captured well using the traditional Poisson or Bernoulli arrival processes, and hence Markovian arrival process (MAP) can be adopted to cope with the bursty and correlated nature of the arrival process, see Chakravarthy [8,9]. In particular, the discrete-time analogue of MAP is discrete-Markovian arrival process (D-MAP) which is more applicable in telecommunication context due to the discrete nature of the transmission of information units in slotted systems, see for example Alfa [2,3], Bruneel and Kim [7], Hunter [19], Takagi [25] and Woodward [26]. The arrival process D-MAP is also a versatile arrival process and covers many other well known arrival processes such as the Bernoulli arrival process, the switched Bernoulli process (SBP), the Markov modulated Bernoulli process (MMBP), and the discrete-time PH-renewal process. Much work has been done in the past on queueing models with D-MAP arrival process with both finite and infinite buffers. As for instance, Chaudhry and Gupta $[10,11]$ studied the finite-buffer D-MAP $/ G / 1 / N$ and D-MAP $/ G^{(a, b)} / 1 / N$ queues where they obtained the queue-content distribution at various epochs. Further, Gupta et al. [17] addressed a more general D-MAP $/ G_{n} / 1 / N$ queue with the service time depending on the number of customers waiting in the queue. Yu and Alfa [27] considered a batch-size dependent service D-MAP $/ G_{n}^{(1, a, b)} / 1 / N$ queue where the server serves the customers individually if there are less than " $a$ " customers in the queue, otherwise it servers according to the general bulk service $(a, b)$ rule. For the infinite-buffer queue, Pradhan and Gupta [22] addressed the continuous-time MAP $/ G_{n}^{(a, b)} / 1$ queue whereas Claeys et al. [14] studied the discrete time analogue of [22] with the assumption of batch Markovian arrival process.

In this paper, we present a complete theoretical and computational analysis of an infinite-buffer discrete-time queueing model with the arrival process as D-MAP. We assume that the server provides service in batches according to the general bulk service rule and the service time follows general distribution and depends on the size of the batch undergoing service. At first, using the supplementary variable technique, we obtain the steadystate bivariate vector generating function (VGF) of the queue-length and server content at the departure epoch of the batch, in a completely known form. Using the bivariate VGF we extract the distribution at the departure epoch in terms of roots of the associated characteristic equation. Further, in order to obtain the distribution at arbitrary, pre-arrival and outside observer's epochs, we establish their relation with the distribution at the departure epoch. Keeping note of the complexity of the model under consideration, we discuss in detail the whole computing process by considering discrete phase-type and arbitrary distributed service time distributions which cover almost all distributions that arise in various applications. We evaluate some significant performance characteristics of the model and demonstrate the computing process by considering certain numerical examples. It may be noted here that the use of supplementary variable technique makes the analysis of the model relatively simpler, which, otherwise, would have been difficult using the embedded Markov chain technique because of the complexity associated with the construction of the transition probability matrix.

It may be mentioned that Claeys et al. [14] have discussed a batch-service queue with batch Markovian arrival process and batch-size-dependent service time i.e., D-BMAP $/ G_{n}^{(l, c)} / 1$ queue. They derived the VGF of joint distribution of queue length and server content at arbitrary slot. However, they did not discuss the 
inversion process for extracting the joint probabilities from that VGF. Instead, they derived the queue content distribution when server is inactive, server processes at suboptimal capacity, number in a served batch, and tail distribution. They also investigated the influence of correlation of the arrival process on the behavior of the system. In the current paper, we first provide the VGF of queue length and server content distribution together at departure or service completion epoch and then present a simple and elegant procedure for extracting the joint probabilities from the VGF which involves several complicated mathematical expressions. We also provide some pertinent marginal distribution along with performance measures using known distributions which are simple and easy to use.

The information about the joint probabilities of queue and server content has notable advantages in many applications to control the congestion at the queue level as well as at the server level. For example, for the transmission of data in the mobile network from a base station (BS) to a suitable client station, where packets of data arrive and are queued if the transmission channel is busy for onward transmission. The packets are transmitted in batches with a minimum and maximum thresholds, and the transmission time is dependent on the batch-size. In the case of heavy load at the BS as well with the transmission channel, onward transmission of packets can be speeded up by knowing both the loads at the queue as well as at the transmission level. The distribution of only either queue content or server content may not be helpful to adequately frame such scenarios. The knowledge about the average number of customers with the server enables us to maximize the serving capability of the server, for which the server content distribution is inescapable. It also plays a noteworthy role to compute the system content distribution.

The remaining portion of the paper is organized as follows. In Section 2, we give the detailed description of the considered discrete-time system followed by the analysis of the model in Section 3. In Section 4, we obtain the joint queue and server content distribution at various epochs and then discuss the detailed computing process in Section 5. In Section 6, we discuss a few special cases of the model and evaluate number of performance measures in Section 7. We present various numerical examples in Section 8 which is followed by the conclusion.

\section{MODEL DESCRIPTION, ASSUMPTIONS AND NOTATIONS}

We consider a discrete-time queueing model in which the customers arrive according to arrival process D-MAP and service time of the batches of customers follow general distribution. Below we describe various processes:

- Arrival process: in D-MAP the arrivals are governed by an underlying $m$-state Markov chain having probability $C_{i j},(1 \leq i, j \leq m)$ with a transition from state $i$ to $j$ without an arrival, and having probability $D_{i j},(1 \leq i, j \leq m)$ with a transition from state $i$ to $j$ with an arrival. Let $\mathbf{C}=\left[C_{i j}\right], \mathbf{D}=\left[D_{i j}\right]$ be the $m \times m$ non-negative matrices both having at least one positive entry. The matrix $(\mathbf{C}+\mathbf{D})$ with $(\mathbf{C}+\mathbf{D}) \boldsymbol{e}=\boldsymbol{e}$, where $\boldsymbol{e}$ is a column vector of ones with suitable dimension, is a stochastic matrix corresponding to an irreducible Markov chain underlying the D-MAP. Let $\bar{\pi}=\left[\bar{\pi}_{1}, \bar{\pi}_{2}, \ldots, \bar{\pi}_{m}\right]$ be the stationary probability vector of the underlying Markov chain implying $\overline{\boldsymbol{\pi}}(\mathbf{C}+\mathbf{D})=\overline{\boldsymbol{\pi}}, \overline{\boldsymbol{\pi}} \boldsymbol{e}=1$. The fundamental stationary arrival rate is given by $\lambda^{*}=\bar{\pi} \mathrm{D} e$.

- Service rule: a single server serves the customers in batches according to general bulk service $(a, b)$ rule. If the queue contains less than " $a$ " customers, server enters into the idle period and waits to initiate the service until at least " $a$ " customers get accumulated. When the queue size is $r(a \leq r \leq b)$, entire group of customers are taken for service. However, if the queue size is greater than " $b$ ", the server serves first " $b$ " customers and the remaining customers have to wait for the next round of service.

- Service process: the service time of the batches follow general distribution and are assumed to be dependent on batch size of the ongoing service. Let us define the random variable $V_{r}(a \leq r \leq b)$ as the service time of a batch of size $r$ with probability mass function $s_{r}(l)=\operatorname{Pr}\left(V_{r}=l\right), l \geq 1$, probability generating function $S_{r}^{*}(z)=\sum_{l=1}^{\infty} s_{r}(l) z^{l}$, and the mean service time $\mu_{r}^{-1}=S_{r}=\sum_{l=1}^{\infty} l s_{r}(l)=S_{r}^{*(1)}(1)$, where $S_{r}^{*(1)}(1)=\left.\frac{\mathrm{d}}{\mathrm{d} z} S_{r}^{*}(z)\right|_{z=1}$. 


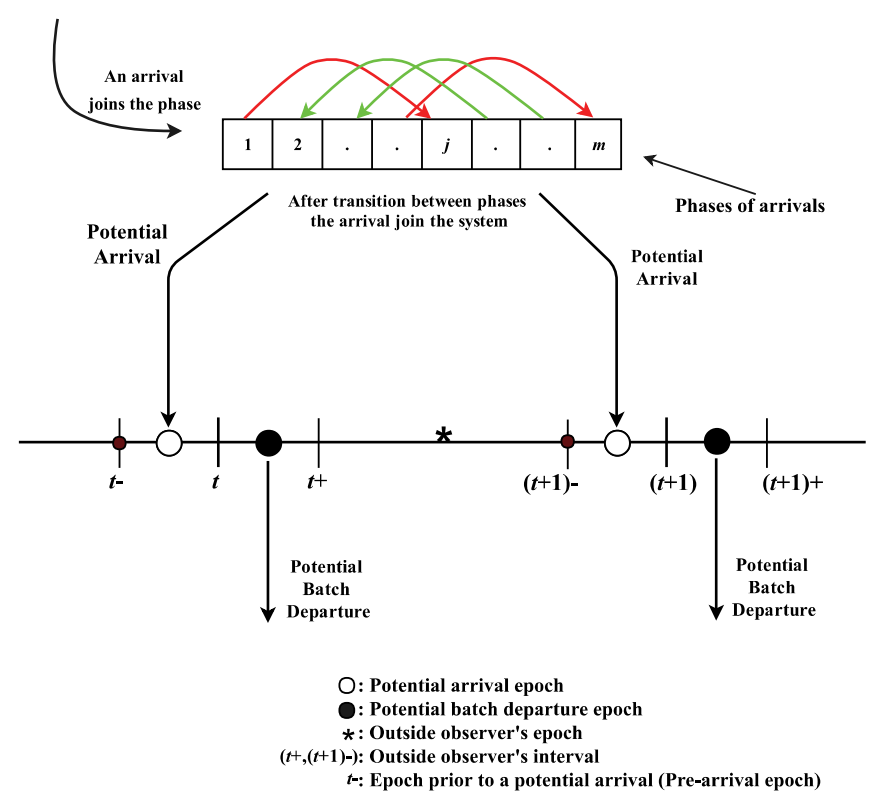

FiguRE 1. Various time epochs in LAS-DA.

- Late arrival system with delayed access: in discrete-time, the time axis is divided into intervals of equal length referred to as (time) slots, separated by slot boundaries. We assume that the length of each slot is unity and time axis is marked as $0,1,2, \ldots, t, \ldots$ We further assume that a potential arrival occurs in the interval $(t-, t)$ and a potential departure takes place in the interval $(t, t+)$. However, if an arrival finds the server idle, it cannot depart in the same slot in which it has arrived and has to wait for at least one slot before getting served. This is referred to as late arrival system with delayed access (LAS-DA), see Hunter [19]. Various time epochs at which events occur are delineated in Figure 1.

- For the stability of the system, we must have that $\rho<1$ where $\rho=\frac{\lambda^{*}}{b \mu_{b}}$.

Let $\mathbf{A}_{r}(n, k), a \leq r \leq b$, be the matrix of order $m \times m$ whose $(i, j)$ th element is the conditional probability that, a departure which left at least $a$ customers in the queue with the arrival process in state $i$, exactly $n$ new customers arrive during the service period (of length $k$ slots) of a batch of $r$ customers; the phase of the arrival process is $j$ at the departure epoch. Thus $\mathbf{A}_{r}(n, k)$ can be written as

$$
\begin{aligned}
& \mathbf{A}_{r}(0, k)=\mathbf{A}_{r}(0, k-1) \mathbf{C}, \quad k \geq 1 \\
& \mathbf{A}_{r}(n, k)=\mathbf{A}_{r}(n, k-1) \mathbf{C}+\mathbf{A}_{r}(n-1, k-1) \mathbf{D}, \quad k \geq n \geq 1,
\end{aligned}
$$

with $\mathbf{A}_{r}(0,0)=\mathbf{I}$ and $\mathbf{A}_{r}(n, k)=\mathbf{0}, n>k \geq 0$, where $\mathbf{I}$ and $\mathbf{0}$ are identity and zero matrix of order $m \times m$, respectively. Also, let $\mathbf{A}_{r}^{*}(z, k)$ be the matrix-generating function of $\mathbf{A}_{r}(n, k)$, then

$$
\mathbf{A}_{r}^{*}(z, k)=\sum_{n=0}^{\infty} \mathbf{A}_{r}(n, k) z^{n}=\left[\mathbf{A}_{r}^{*}(z, 1)\right]^{k}=[\mathbf{C}+\mathbf{D} z]^{k},
$$

where $\mathbf{A}_{r}^{*}(z, 1)=\mathbf{C}+\mathbf{D} z$ is the matrix-generating function of the number of customers arriving in one slot. Let us denote the matrix $\mathbf{A}_{r}(n)=\left[A_{r}(n)\right]_{i, j}, n \geq 0, a \leq r \leq b, 1 \leq i, j \leq m$, to be the conditional probability that, a departure left at least " $a$ " customers in the queue with the arrival process in state $i$, and during the service period of $r$ customers exactly $n$ new customers arrive with the phase of the arrival process being $j$ at 
the departure epoch. So, $\mathbf{A}_{r}(n)$ can be written as

$$
\mathbf{A}_{r}(n)=\sum_{k=\max (1, n)}^{\infty} s_{r}(k) \mathbf{A}_{r}(n, k), \quad n \geq 0 .
$$

Further, let $\mathbf{A}_{r}^{*}(z)$ be the matrix-generating function of $\mathbf{A}_{r}(n)$. Therefore, we have

$$
\mathbf{A}_{r}^{*}(z)=\sum_{n=0}^{\infty} \mathbf{A}_{r}(n) z^{n}=\sum_{k=1}^{\infty} s_{r}(k)[\mathbf{C}+\mathbf{D} z]^{k} \equiv S_{r}(\mathbf{C}+\mathbf{D} z), \quad a \leq r \leq b .
$$

Remark 2.1. As and when more notations are used, they are defined at respective places.

\section{Analysis of the MOdel}

Let us define the following random variables at the beginning of the slot boundary i.e. just before a potential arrival:

- $N_{q}(t-) \equiv$ Number of customers in the queue waiting for service at $t-$.

$-N_{s}(t-) \equiv$ Number of customers with the server at $t-$.

$-J(t-) \equiv$ Phase of the arrival process at $t-$.

$-U(t-) \equiv$ Remaining service time of a batch in service (if any) at $t-$.

Let us define for $1 \leq i \leq m$,

$$
\begin{array}{r}
p_{i}(n, 0 ; t-)=\operatorname{Pr}\left\{N_{q}(t-)=n, N_{s}(t-)=0, J(t-)=i, \text { server idle }\right\}, \quad 0 \leq n \leq a-1 \\
\pi_{i}(n, r, u ; t-)=\operatorname{Pr}\left\{N_{q}(t-)=n, N_{s}(t-)=r, J(t-)=i, U(t-)=u, \text { server busy }\right\} \\
n \geq 0, a \leq r \leq b, u \geq 1
\end{array}
$$

Also, let us define the limiting probabilities as

$$
\begin{aligned}
p_{i}(n, 0) & =\lim _{t-\rightarrow \infty} p_{i}(n, 0 ; t-), \\
\pi_{i}(n, r, u) & =\lim _{t-\rightarrow \infty} \pi_{i}(n, r, u ; t-) .
\end{aligned}
$$

Further, we define

$$
\begin{aligned}
\mathbf{p}(n, 0) & =\left[p_{1}(n, 0), \ldots, p_{i}(n, 0), \ldots, p_{m}(n, 0)\right], \\
\boldsymbol{\pi}(n, r, u) & =\left[\pi_{1}(n, r, u), \ldots, \pi_{i}(n, r, u), \ldots, \pi_{m}(n, r, u)\right] .
\end{aligned}
$$

Relating the states of the system at two consecutive time epochs $t-$ and $(t+1)$ - for each phase, and then using matrix and vector notations after taking $t-\rightarrow \infty$, we obtain, in steady-state,

$$
\begin{aligned}
\mathbf{p}(0,0)= & \mathbf{p}(0,0) \mathbf{C}+\sum_{r=a}^{b} \boldsymbol{\pi}(0, r, 1) \mathbf{C}, \\
\mathbf{p}(n, 0)= & \mathbf{p}(n, 0) \mathbf{C}+\sum_{r=a}^{b} \boldsymbol{\pi}(n, r, 1) \mathbf{C}+\mathbf{p}(n-1,0) \mathbf{D}+\sum_{r=a}^{b} \boldsymbol{\pi}(n-1, r, 1) \mathbf{D}, \\
& 1 \leq n \leq a-1,
\end{aligned}
$$




$$
\begin{aligned}
\boldsymbol{\pi}(0, a, u)= & \boldsymbol{\pi}(0, a, u+1) \mathbf{C}+\mathbf{p}(a-1,0) \mathbf{D} s_{a}(u)+\sum_{r=a}^{b} \boldsymbol{\pi}(a, r, 1) \mathbf{C} s_{a}(u) \\
& +\sum_{r=a}^{b} \boldsymbol{\pi}(a-1, r, 1) \mathbf{D} s_{a}(u), \\
\boldsymbol{\pi}(0, r, u)= & \boldsymbol{\pi}(0, r, u+1) \mathbf{C}+\sum_{i=a}^{b} \boldsymbol{\pi}(r, i, 1) \mathbf{C} s_{r}(u)+\sum_{i=a}^{b} \boldsymbol{\pi}(r-1, i, 1) \mathbf{D} s_{r}(u), \\
& a+1 \leq r \leq b, \\
\boldsymbol{\pi}(n, r, u)= & \boldsymbol{\pi}(n, r, u+1) \mathbf{C}+\boldsymbol{\pi}(n-1, r, u+1) \mathbf{D}, \quad n \geq 1, a \leq r \leq b-1, \\
\boldsymbol{\pi}(n, b, u)= & \boldsymbol{\pi}(n, b, u+1) \mathbf{C}+\boldsymbol{\pi}(n-1, b, u+1) \mathbf{D}+\sum_{r=a}^{b} \boldsymbol{\pi}(n+b, r, 1) \mathbf{C} s_{b}(u) \\
& +\sum_{r=a}^{b} \boldsymbol{\pi}(n+b-1, r, 1) \mathbf{D} s_{b}(u), \quad n \geq 1 .
\end{aligned}
$$

Let us define the VGF of $\boldsymbol{\pi}(n, r, u)$ as

$$
\boldsymbol{\pi}^{*}(n, r, z)=\sum_{u=1}^{\infty} \boldsymbol{\pi}(n, r, u) z^{u}, \quad|z| \leq 1, n \geq 0, a \leq r \leq b .
$$

It follows from (3.7) that

$$
\boldsymbol{\pi}^{*}(n, r, 1)=\sum_{u=1}^{\infty} \boldsymbol{\pi}(n, r, u)=\boldsymbol{\pi}(n, r), \quad n \geq 0, a \leq r \leq b .
$$

Multiplying (3.3)-(3.6) by $z^{u}$ and summing over $u$ from 1 to $\infty$, we get

$$
\begin{aligned}
\boldsymbol{\pi}^{*}(0, a, z)= & \frac{1}{z}\left(\boldsymbol{\pi}^{*}(0, a, z)-z \boldsymbol{\pi}(0, a, 1)\right) \mathbf{C}+\mathbf{p}(a-1,0) \mathbf{D} S_{a}^{*}(z) \\
& +\sum_{r=a}^{b} \boldsymbol{\pi}(a, r, 1) \mathbf{C} S_{a}^{*}(z)+\sum_{r=a}^{b} \boldsymbol{\pi}(a-1, r, 1) \mathbf{D} S_{a}^{*}(z), \\
\boldsymbol{\pi}^{*}(0, r, z)= & \frac{1}{z}\left(\boldsymbol{\pi}^{*}(0, r, z)-z \boldsymbol{\pi}(0, r, 1)\right) \mathbf{C}+\sum_{i=a}^{b} \boldsymbol{\pi}(r, i, 1) \mathbf{C} S_{r}^{*}(z) \\
& +\sum_{i=a}^{b} \boldsymbol{\pi}(r-1, i, 1) \mathbf{D} S_{r}^{*}(z), \quad a+1 \leq r \leq b, \\
\boldsymbol{\pi}^{*}(n, r, z)= & \frac{1}{z}\left(\boldsymbol{\pi}^{*}(n, r, z)-z \boldsymbol{\pi}(n, r, 1)\right) \mathbf{C} \\
& +\frac{1}{z}\left(\boldsymbol{\pi}^{*}(n-1, r, z)-z \boldsymbol{\pi}(n-1, r, 1)\right) \mathbf{D}, \quad n \geq 1, a \leq r \leq b-1, \\
\boldsymbol{\pi}^{*}(n, b, z)= & \frac{1}{z}\left(\boldsymbol{\pi}^{*}(n, b, z)-z \boldsymbol{\pi}(n, b, 1)\right) \mathbf{C}+\frac{1}{z}\left(\boldsymbol{\pi}^{*}(n-1, b, z)-z \boldsymbol{\pi}(n-1, b, 1)\right) \mathbf{D} \\
& +\sum_{r=a}^{b} \boldsymbol{\pi}(n+b, r, 1) \mathbf{C} S_{b}^{*}(z)+\sum_{r=a}^{b} \boldsymbol{\pi}(n+b-1, r, 1) \mathbf{D} S_{b}^{*}(z), \quad n \geq 1 .
\end{aligned}
$$


Post multiplying (3.1) and (3.2) by $\boldsymbol{e}$ and then summing over $n$ and $r$, we get

$$
\mathbf{p}(a-1,0) \mathbf{D} \boldsymbol{e}=\sum_{n=0}^{a-1} \sum_{r=a}^{b} \boldsymbol{\pi}(n, r, 1) \mathbf{C} \boldsymbol{e}+\sum_{n=0}^{a-2} \sum_{r=a}^{b} \boldsymbol{\pi}(n, r, 1) \mathbf{D} \boldsymbol{e} .
$$

Post multiplying (3.8)-(3.11) by $\boldsymbol{e}$ and then summing over $n$ and $r$, we get

$$
\begin{aligned}
\left(\frac{z-1}{z}\right) \sum_{n=0}^{\infty} \sum_{r=a}^{b} \boldsymbol{\pi}^{*}(n, r, z) \boldsymbol{e}= & -\sum_{n=0}^{\infty} \sum_{r=a}^{b} \boldsymbol{\pi}(n, r, 1) \boldsymbol{e} \\
& +\sum_{n=0}^{a-1} \sum_{r=a}^{b} \boldsymbol{\pi}(n, r, 1) \mathbf{C} \boldsymbol{e} S_{a}^{*}(z)+\sum_{n=0}^{a-2} \sum_{r=a}^{b} \boldsymbol{\pi}(n, r, 1) \mathbf{D} \boldsymbol{e} S_{a}^{*}(z) \\
& +\sum_{r=a}^{b} \boldsymbol{\pi}(a, r, 1) \mathbf{C} \boldsymbol{e} S_{a}^{*}(z)+\sum_{r=a}^{b} \boldsymbol{\pi}(a-1, r, 1) \mathbf{D} \boldsymbol{e} S_{a}^{*}(z), \\
& +\sum_{n=a+1}^{b} \sum_{r=a}^{b} \boldsymbol{\pi}(n, r, 1) \mathbf{C} S_{n}^{*}(z)+\sum_{n=a}^{b-1} \sum_{r=a}^{b} \boldsymbol{\pi}(n, r, 1) \mathbf{D} \boldsymbol{e} S_{n}^{*}(z) \\
& +\sum_{n=b+1}^{\infty} \sum_{r=a}^{b} \boldsymbol{\pi}(n, r, 1) \mathbf{C} \boldsymbol{e} S_{b}^{*}(z)+\sum_{n=b}^{\infty} \sum_{r=a}^{b} \boldsymbol{\pi}(n, r, 1) \mathbf{D} \boldsymbol{e} S_{b}^{*}(z) \\
\Rightarrow\left(\frac{z-1}{z}\right) \sum_{n=0}^{\infty} \sum_{r=a}^{b} \boldsymbol{\pi}^{*}(n, r, z) \boldsymbol{e}= & \sum_{n=0}^{a} \sum_{r=a}^{b} \boldsymbol{\pi}(n, r, 1) \mathbf{C e}\left(S_{a}^{*}(z)-1\right)+\sum_{n=0}^{a-1} \sum_{r=a}^{b} \boldsymbol{\pi}(n, r, 1) \mathbf{D} \boldsymbol{e}\left(S_{a}^{*}(z)-1\right) \\
& +\sum_{n=a+1}^{b} \sum_{r=a}^{b} \boldsymbol{\pi}(n, r, 1) \mathbf{C} \boldsymbol{e}\left(S_{n}^{*}(z)-1\right)+\sum_{n=a}^{b-1} \sum_{r=a}^{b} \boldsymbol{\pi}(n, r, 1) \mathbf{D} \boldsymbol{e}\left(S_{n}^{*}(z)-1\right) \\
& +\sum_{n=b+1}^{\infty} \sum_{r=a}^{b} \boldsymbol{\pi}(n, r, 1) \mathbf{C} \boldsymbol{e}\left(S_{b}^{*}(z)-1\right)+\sum_{n=b}^{\infty} \sum_{r=a}^{b} \boldsymbol{\pi}(n, r, 1) \mathbf{D} \boldsymbol{e}\left(S_{b}^{*}(z)-1\right) .
\end{aligned}
$$

Letting $z \rightarrow 1$ in (3.14), we get

$$
\begin{aligned}
1-\sum_{n=0}^{a-1} \mathbf{p}(n, 0) \boldsymbol{e}= & \sum_{n=0}^{a} \sum_{r=a}^{b} \boldsymbol{\pi}(n, r, 1) \boldsymbol{e} S_{a}+\sum_{n=a+1}^{b} \sum_{r=a}^{b} \boldsymbol{\pi}(n, r, 1) \boldsymbol{e} S_{n} \\
& +\sum_{n=b+1}^{\infty} \sum_{r=a}^{b} \boldsymbol{\pi}(n, r, 1) \boldsymbol{e} S_{b} .
\end{aligned}
$$

Further, define the VGF of $\boldsymbol{\pi}^{*}(n, r, z)$ as

$$
\tilde{\boldsymbol{\pi}}^{*}(x, \xi, z)=\sum_{n=0}^{\infty} \sum_{r=a}^{b} \boldsymbol{\pi}^{*}(n, r, z) x^{n} \xi^{r}, \quad|x| \leq 1,|\xi| \leq 1,|z| \leq 1 .
$$

Now multiplying (3.8)-(3.11) by $x^{n}$ and $\xi^{r}$ and summing over $n$ from 0 to $\infty$, and $r$ from $a$ to $b$, we get

$$
\tilde{\boldsymbol{\pi}}^{*}(x, \xi, z)\left(\frac{z \mathbf{I}-\mathbf{C}-\mathbf{D} x}{z}\right)=-\sum_{n=0}^{\infty} \sum_{r=a}^{b} \boldsymbol{\pi}(n, r, 1) x^{n} \xi^{r}(\mathbf{C}+\mathbf{D} x)+\mathbf{p}(a-1,0) \mathbf{D} \xi^{a} S_{a}^{*}(z)
$$




$$
\begin{aligned}
& +\sum_{n=a}^{b} \sum_{r=a}^{b}(\boldsymbol{\pi}(n, r, 1) \mathbf{C}+\boldsymbol{\pi}(n-1, r, 1) \mathbf{D}) \xi^{n} S_{n}^{*}(z) \\
& +\sum_{n=b+1}^{\infty} \sum_{r=a}^{b}(\boldsymbol{\pi}(n, r, 1) \mathbf{C}+\boldsymbol{\pi}(n-1, r, 1) \mathbf{D}) x^{n-b} \xi^{b} S_{b}^{*}(z)
\end{aligned}
$$

Our aim is to determine the bivariate VGF of the queue and server content. For this, we utilize the eigenvalues and eigenvectors of $(\mathbf{C}+\mathbf{D} x)$, see Claeys et al. [14] and Pradhan and Gupta [22]. Now, let $\gamma_{1}(x), \gamma_{2}(x), \ldots, \gamma_{m}(x)$ be the eigenvalues and $\boldsymbol{\eta}_{\mathbf{1}}(x), \boldsymbol{\eta}_{\mathbf{2}}(x), \ldots, \boldsymbol{\eta}_{\boldsymbol{m}}(x)$ be the corresponding right eigenvectors of $(\mathbf{C}+\mathbf{D} x)$. Thus, for $1 \leq i \leq m$, we have

$$
\begin{aligned}
(\mathbf{C}+\mathbf{D} x) \boldsymbol{\eta}_{\boldsymbol{i}}(x) & =\gamma_{i}(x) \boldsymbol{\eta}_{\boldsymbol{i}}(x), \\
\left\{\gamma_{i}(x) \mathbf{I}-(\mathbf{C}+\mathbf{D} x)\right\} \boldsymbol{\eta}_{\boldsymbol{i}}(x) & =\mathbf{0} .
\end{aligned}
$$

Setting $z=\gamma_{i}(x)$ in (3.16) and post-multiplying it by $\boldsymbol{\eta}_{\boldsymbol{i}}(x)$ on both sides and using (3.17), we get

$$
\begin{aligned}
\sum_{n=0}^{\infty} \sum_{r=a}^{b} \boldsymbol{\pi}(n, r, 1) x^{n} \xi^{r}(\mathbf{C}+\mathbf{D} x) \boldsymbol{\eta}_{\boldsymbol{i}}(x)= & \mathbf{p}(a-1,0) \mathbf{D} \xi^{a} S_{a}^{*}\left(\gamma_{i}(x)\right) \boldsymbol{\eta}_{\boldsymbol{i}}(x) \\
& +\sum_{n=a}^{b} \sum_{r=a}^{b}(\boldsymbol{\pi}(n, r, 1) \mathbf{C}+\boldsymbol{\pi}(n-1, r, 1) \mathbf{D}) \xi^{n} S_{n}^{*}\left(\gamma_{i}(x)\right) \boldsymbol{\eta}_{\boldsymbol{i}}(x) \\
& +\sum_{n=b+1}^{\infty} \sum_{r=a}^{b}(\boldsymbol{\pi}(n, r, 1) \mathbf{C}+\boldsymbol{\pi}(n-1, r, 1) \mathbf{D}) x^{n-b} \xi^{b} S_{b}^{*}\left(\gamma_{i}(x)\right) \boldsymbol{\eta}_{\boldsymbol{i}}(x) .
\end{aligned}
$$

Since (3.18) is true for all $i$ from 1 to $m$, so we have

$$
\begin{aligned}
\sum_{n=0}^{\infty} \sum_{r=a}^{b} \boldsymbol{\pi}(n, & r, 1) x^{n} \xi^{r}(\mathbf{C}+\mathbf{D} x)\left[\boldsymbol{\eta}_{\mathbf{1}}(x), \ldots, \boldsymbol{\eta}_{\boldsymbol{m}}(x)\right] \\
= & \mathbf{p}(a-1,0) \mathbf{D} \xi^{a}\left[S_{a}^{*}\left(\gamma_{1}(x)\right) \boldsymbol{\eta}_{\mathbf{1}}(x), \ldots, S_{a}^{*}\left(\gamma_{m}(x)\right) \boldsymbol{\eta}_{\boldsymbol{m}}(x)\right] \\
& +\sum_{n=a}^{b} \sum_{r=a}^{b}(\boldsymbol{\pi}(n, r, 1) \mathbf{C}+\boldsymbol{\pi}(n-1, r, 1) \mathbf{D}) \xi^{n}\left[S_{n}^{*}\left(\gamma_{1}(x)\right) \boldsymbol{\eta}_{\mathbf{1}}(x), \ldots, S_{n}^{*}\left(\gamma_{m}(x)\right) \boldsymbol{\eta}_{\boldsymbol{m}}(x)\right] \\
& +\sum_{n=b+1}^{\infty} \sum_{r=a}^{b}(\boldsymbol{\pi}(n, r, 1) \mathbf{C}+\boldsymbol{\pi}(n-1, r, 1) \mathbf{D}) x^{n-b} \xi^{b}\left[S_{b}^{*}\left(\gamma_{1}(x)\right) \boldsymbol{\eta}_{\mathbf{1}}(x), \ldots, S_{b}^{*}\left(\gamma_{m}(x)\right) \boldsymbol{\eta}_{\boldsymbol{m}}(x)\right] .
\end{aligned}
$$

Further, define

$$
\mathbf{R}(x)=\left[\boldsymbol{\eta}_{\mathbf{1}}(x), \boldsymbol{\eta}_{\mathbf{2}}(x), \ldots, \boldsymbol{\eta}_{\boldsymbol{m}}(x)\right]
$$

The inverse of $\mathbf{R}(x)$ exists whenever each eigenvalue is of multiplicity 1. For details, see [14, 22]. Now, using (3.20) in (3.19) and then post-multiplying it by $\mathbf{R}^{-1}(x)$, we get

$$
\begin{aligned}
\sum_{n=0}^{\infty} \sum_{r=a}^{b} \boldsymbol{\pi}(n, r, 1) x^{n} \xi^{r}(\mathbf{C}+\mathbf{D} x) & \\
= & \mathbf{p}(a-1,0) \mathbf{D} \xi^{a} \mathbf{R}(x)\left[\operatorname{diag}\left\{S_{a}^{*}\left(\gamma_{i}(x)\right)\right\}_{i=1}^{m}\right] \mathbf{R}^{-1}(x) \\
& +\sum_{n=a}^{b} \sum_{r=a}^{b}(\boldsymbol{\pi}(n, r, 1) \mathbf{C}+\boldsymbol{\pi}(n-1, r, 1) \mathbf{D}) \xi^{n} \mathbf{R}(x)\left[\operatorname{diag}\left\{S_{n}^{*}\left(\gamma_{i}(x)\right)\right\}_{i=1}^{m}\right] \mathbf{R}^{-1}(x)
\end{aligned}
$$




$$
+\sum_{n=b+1}^{\infty} \sum_{r=a}^{b}(\boldsymbol{\pi}(n, r, 1) \mathbf{C}+\boldsymbol{\pi}(n-1, r, 1) \mathbf{D}) x^{n-b} \xi^{b} \mathbf{R}(x)\left[\operatorname{diag}\left\{S_{b}^{*}\left(\gamma_{i}(x)\right)\right\}_{i=1}^{m}\right] \mathbf{R}^{-1}(x),
$$

where $\left[\operatorname{diag}\left\{S_{r}^{*}\left(\gamma_{i}(x)\right)\right\}_{i=1}^{m}\right], \quad a \leq r \leq b$, is a diagonal matrix of order $m$ with diagonal entries $S_{r}^{*}\left(\gamma_{1}(x)\right), \ldots, S_{r}^{*}\left(\gamma_{m}(x)\right)$. Using the theory of eigenvalues and eigenvectors, we can write

$$
\begin{aligned}
& \mathbf{C}+\mathbf{D} x=\mathbf{R}(x)\left[\operatorname{diag}\left\{\gamma_{i}(x)\right\}_{i=1}^{m}\right] \mathbf{R}^{-1}(x), \\
& \text { and } \quad S_{r}^{*}(\mathbf{C}+\mathbf{D} x)=\mathbf{R}(x)\left[\operatorname{diag}\left\{S_{r}^{*}\left(\gamma_{i}(x)\right)\right\}_{i=1}^{m}\right] \mathbf{R}^{-1}(x) \text {. }
\end{aligned}
$$

Now, using (2.2) and (3.22) in (3.21), we obtain

$$
\begin{aligned}
\sum_{n=0}^{\infty} \sum_{r=a}^{b} \boldsymbol{\pi}(n, r, 1) x^{n} \xi^{r}(\mathbf{C}+\mathbf{D} x)= & \mathbf{p}(a-1,0) \mathbf{D} \xi^{a} \mathbf{A}_{a}^{*}(x) \\
& +\sum_{n=a}^{b} \sum_{r=a}^{b}(\boldsymbol{\pi}(n, r, 1) \mathbf{C}+\boldsymbol{\pi}(n-1, r, 1) \mathbf{D}) \xi^{n} \mathbf{A}_{n}^{*}(x) \\
& +\sum_{n=b+1}^{\infty} \sum_{r=a}^{b}(\boldsymbol{\pi}(n, r, 1) \mathbf{C}+\boldsymbol{\pi}(n-1, r, 1) \mathbf{D}) x^{n-b} \xi^{b} \mathbf{A}_{b}^{*}(x),
\end{aligned}
$$

where $\mathbf{A}_{r}^{*}(x)=S_{r}^{*}(\mathbf{C}+\mathbf{D} x),(a \leq r \leq b)$. Now, using (3.23), we obtain the bivariate VGF of the queue content at the departure epoch which is given in the next section.

\subsection{Bivariate VGF at departure epoch}

Let us define $\pi^{+}(n, r)=\left[\pi_{1}^{+}(n, r), \pi_{2}^{+}(n, r), \ldots, \pi_{m}^{+}(n, r)\right]$ as the joint probability vector whose $j$ th element $\left(\pi_{j}^{+}(n, r)\right)$ is the probability that there are $n$ customers in the queue at departure epoch of a batch of size $r$ and arrival process is in phase $j$. Also let $\phi^{+}(n)=$ Probability vector that there are $n$ customers in the queue at departure epoch of a batch $=\sum_{r=a}^{b} \boldsymbol{\pi}^{+}(n, r)$. Further, define $\tilde{\boldsymbol{\pi}}^{+}(x, \xi)=\sum_{n=0}^{\infty} \sum_{r=a}^{b} \boldsymbol{\pi}^{+}(n, r) x^{n} \xi^{r}$ and $\boldsymbol{\Phi}^{+}(x)=\sum_{n=0}^{\infty} \boldsymbol{\phi}^{+}(n) x^{n}$. Using probabilistic arguments, $\boldsymbol{\pi}^{+}(n, r)$ and $\boldsymbol{\pi}(n, r, 1)$ are connected as:

$$
\begin{aligned}
& \boldsymbol{\pi}^{+}(0, r)=\Omega \boldsymbol{\pi}(0, r, 1) \mathbf{C} \\
& \boldsymbol{\pi}^{+}(n, r)=\Omega(\boldsymbol{\pi}(n, r, 1) \mathbf{C}+\boldsymbol{\pi}(n-1, r, 1) \mathbf{D}), n \geq 1, a \leq r \leq b,
\end{aligned}
$$

where $\Omega^{-1}=\sum_{n=0}^{\infty} \sum_{r=a}^{b} \boldsymbol{\pi}(n, r, 1) \boldsymbol{e}$.

\section{Lemma 3.1.}

$$
\Omega^{-1}=\psi^{-1}\left(1-\sum_{n=0}^{a-1} \mathbf{p}(n, 0) \boldsymbol{e}\right)
$$

where $\psi=\sum_{n=0}^{a-1} \phi^{+}(n) e S_{a}+\sum_{n=a}^{b} \phi^{+}(n) e S_{n}+\sum_{n=b+1}^{\infty} \phi^{+}(n) e S_{b}$.

Proof. Equation (3.15) can be written as

$$
\begin{aligned}
1-\sum_{n=0}^{a-1} \mathbf{p}(n, 0) \boldsymbol{e}= & \sum_{r=a}^{b} \boldsymbol{\pi}(0, r, 1) \mathbf{C} \boldsymbol{e} S_{a}+\sum_{n=1}^{a-1} \sum_{r=a}^{b}(\boldsymbol{\pi}(n, r, 1) \mathbf{C}+\boldsymbol{\pi}(n-1, r, 1) \mathbf{D}) \boldsymbol{e} S_{a} \\
& +\sum_{n=a}^{b} \sum_{r=a}^{b}(\boldsymbol{\pi}(n, r, 1) \mathbf{C}+\boldsymbol{\pi}(n-1, r, 1) \mathbf{D}) \boldsymbol{e} S_{n}
\end{aligned}
$$




$$
+\sum_{n=b+1}^{\infty} \sum_{r=a}^{b}(\boldsymbol{\pi}(n, r, 1) \mathbf{C}+\boldsymbol{\pi}(n-1, r, 1) \mathbf{D}) \boldsymbol{e} S_{b}
$$

Multiplying both sides of above equation by $\Omega$ and using (3.24) and (3.25), we get

$$
\Omega\left(1-\sum_{n=0}^{a-1} \mathbf{p}(n, 0) \boldsymbol{e}\right)=\sum_{n=0}^{a-1} \boldsymbol{\phi}^{+}(n) \boldsymbol{e} S_{a}+\sum_{n=a}^{b} \boldsymbol{\phi}^{+}(n) \boldsymbol{e} S_{n}+\sum_{n=b+1}^{\infty} \boldsymbol{\phi}^{+}(n) \boldsymbol{e} S_{b} .
$$

\section{Lemma 3.2.}

$$
\Omega \mathbf{p}(a-1,0)=\sum_{n=0}^{a-1} \phi^{+}(n)(\overline{\mathbf{D}})^{(a-1-n)}(\mathbf{I}-\mathbf{C})^{-1}
$$

where $\overline{\mathbf{D}}=(\mathbf{I}-\mathbf{C})^{-1} \mathbf{D}$.

Proof. Multiplying (3.1) by $\Omega$ and using (3.24) and (3.25), we get

$$
\begin{aligned}
\Omega \mathbf{p}(0,0)(\mathbf{I}-\mathbf{C}) & =\phi^{+}(0), \\
\Omega \mathbf{p}(0,0) & =\phi^{+}(0)(\mathbf{I}-\mathbf{C})^{-1} .
\end{aligned}
$$

Now multiplying (3.2) by $\Omega$ for $n=i$ and after simplification, we finally get

$$
\Omega \mathbf{p}(i, 0)(\mathbf{I}-\mathbf{C})=\sum_{n=0}^{i} \phi^{+}(n)(\overline{\mathbf{D}})^{(i-n)}, \quad 1 \leq i \leq a-1,
$$

which gives

$$
\begin{aligned}
\Omega \mathbf{p}(a-1,0)(\mathbf{I}-\mathbf{C}) & =\sum_{n=0}^{a-1} \phi^{+}(n)(\overline{\mathbf{D}})^{(a-1-n)}, \\
\Omega \mathbf{p}(a-1,0) & =\sum_{n=0}^{a-1} \phi^{+}(n)(\overline{\mathbf{D}})^{(a-1-n)}(\mathbf{I}-\mathbf{C})^{-1} .
\end{aligned}
$$

Now multiplying (3.23) by $\Omega$ and using the definition of departure epoch probabilities and Lemma 3.1, we get

$$
\tilde{\boldsymbol{\pi}}^{+}(x, \xi)=\sum_{n=0}^{a-1} \boldsymbol{\phi}^{+}(n)(\overline{\mathbf{D}})^{(a-n)} \xi^{a} \mathbf{A}_{a}^{*}(x)+\sum_{n=a}^{b} \boldsymbol{\phi}^{+}(n) \xi^{n} \mathbf{A}_{n}^{*}(x)+\frac{\xi^{b}}{x^{b}} \sum_{n=b+1}^{\infty} \boldsymbol{\phi}^{+}(n) x^{n} \mathbf{A}_{b}^{*}(x) .
$$

Setting $\xi=1$ in (3.26), we get the VGF of only queue length distribution $\boldsymbol{\Phi}^{+}(x)\left(=\tilde{\boldsymbol{\pi}}^{+}(x, 1)\right)$ as

$$
\boldsymbol{\Phi}^{+}(x)=\sum_{n=0}^{a-1} \phi^{+}(n)(\overline{\mathbf{D}})^{(a-n)} \mathbf{A}_{a}^{*}(x)+\sum_{n=a}^{b} \boldsymbol{\phi}^{+}(n) \mathbf{A}_{n}^{*}(x)+\frac{1}{x^{b}} \sum_{n=b+1}^{\infty} \phi^{+}(n) x^{n} \mathbf{A}_{b}^{*}(x),
$$

which gives

$$
\mathbf{\Phi}^{+}(x)\left(x^{b} \mathbf{I}-\mathbf{A}_{b}^{*}(x)\right)=\sum_{n=0}^{a-1} \boldsymbol{\phi}^{+}(n)\left((\overline{\mathbf{D}})^{(a-n)} x^{b} \mathbf{A}_{a}^{*}(x)-x^{n} \mathbf{A}_{b}^{*}(x)\right)
$$




$$
+\sum_{n=a}^{b-1} \boldsymbol{\phi}^{+}(n)\left(x^{b} \mathbf{A}_{n}^{*}(x)-x^{n} \mathbf{A}_{b}^{*}(x)\right) .
$$

From (3.26), we have

$$
\begin{aligned}
\tilde{\boldsymbol{\pi}}^{+}(x, \xi)= & \sum_{n=0}^{a-1} \boldsymbol{\phi}^{+}(n)(\overline{\mathbf{D}})^{(a-n)} \xi^{a} \mathbf{A}_{a}^{*}(x)+\sum_{n=a}^{b} \boldsymbol{\phi}^{+}(n) \xi^{n} \mathbf{A}_{n}^{*}(x) \\
& +\frac{\xi^{b}}{x^{b}}\left(\boldsymbol{\Phi}^{+}(x)-\sum_{n=0}^{b} \boldsymbol{\phi}^{+}(n) x^{n}\right) \mathbf{A}_{b}^{*}(x) .
\end{aligned}
$$

Now substituting the value of the vector $\boldsymbol{\Phi}^{+}(x)$ from (3.28) into (3.29), we get

$$
\begin{aligned}
\tilde{\boldsymbol{\pi}}^{+}(x, \xi)= & \sum_{n=0}^{a-1} \boldsymbol{\phi}^{+}(n)(\overline{\mathbf{D}})^{(a-n)} \xi^{a} \mathbf{A}_{a}^{*}(x)+\sum_{n=a}^{b} \boldsymbol{\phi}^{+}(n) \xi^{n} \mathbf{A}_{n}^{*}(x) \\
& +\frac{\xi^{b}}{x^{b}}\left(\left[\sum_{n=0}^{a-1} \boldsymbol{\phi}^{+}(n)\left((\overline{\mathbf{D}})^{(a-n)} x^{b} \mathbf{A}_{a}^{*}(x)-x^{n} \mathbf{A}_{b}^{*}(x)\right)\right.\right. \\
& \left.+\sum_{n=a}^{b-1} \boldsymbol{\phi}^{+}(n)\left(x^{b} \mathbf{A}_{n}^{*}(x)-x^{n} \mathbf{A}_{b}^{*}(x)\right)\right]\left(x^{b} \mathbf{I}-\mathbf{A}_{b}^{*}(x)\right)^{-1} \\
& \left.-\sum_{n=0}^{b} \boldsymbol{\phi}^{+}(n) x^{n}\right) \mathbf{A}_{b}^{*}(x) .
\end{aligned}
$$

Post multiplying by $\left(x^{b} \mathbf{I}-\mathbf{A}_{b}^{*}(x)\right)$ on both sides of (3.30), we obtain

$$
\begin{aligned}
\tilde{\boldsymbol{\pi}}^{+}(x, \xi)= & \left(\sum_{n=0}^{a-1} \boldsymbol{\phi}^{+}(n)\left(\left(\xi^{b}-\xi^{a}\right)(\overline{\mathbf{D}})^{(a-n)} \mathbf{A}_{a}^{*}(x) \mathbf{A}_{b}^{*}(x)+(\overline{\mathbf{D}})^{(a-n)} \xi^{a} x^{b} \mathbf{A}_{a}^{*}(x)-\xi^{b} x^{n} \mathbf{A}_{b}^{*}(x)\right)\right. \\
& \left.+\sum_{n=a}^{b-1} \phi^{+}(n)\left(\left(\xi^{b}-\xi^{n}\right) \mathbf{A}_{n}^{*}(x) \mathbf{A}_{b}^{*}(x)+\xi^{n} x^{b} \mathbf{A}_{n}^{*}(x)-\xi^{b} x^{n} \mathbf{A}_{b}^{*}(x)\right)\right)\left(x^{b} \mathbf{I}-\mathbf{A}_{b}^{*}(x)\right)^{-1}
\end{aligned}
$$

\section{JOINT QUEUE AND SERVER CONTENT DISTRIBUTIONS AT VARIOUS EPOCHS}

In this section, we obtain joint queue and server content distribution at arbitrary, pre-arrival and outside observer's epochs.

\subsection{Joint queue and server content distribution at arbitrary epoch}

The joint distribution of queue and server content at arbitrary epoch plays an important role in obtaining system length distribution and also in evaluation of several key performance measures of the queueing model under consideration. The following theorem presents a correspondence between departure and arbitrary epoch probability vectors.

Theorem 4.1. The steady-state probability vectors $\{\mathbf{p}(n, 0), \boldsymbol{\pi}(n, r)\}$ and $\left\{\boldsymbol{\pi}^{+}(n, r), \boldsymbol{\phi}^{+}(n)\right\}$ are connected by

$$
\begin{array}{ll}
\mathbf{p}(n, 0)=\Omega^{-1} \sum_{j=0}^{n} \phi^{+}(j)(\overline{\mathbf{D}})^{(n-j)}(\mathbf{I}-\mathbf{C})^{-1}, & 0 \leq n \leq a-1, \\
\boldsymbol{\pi}(0, a)=\left(\mathbf{p}(a-1,0) \mathbf{D}+\Omega^{-1}\left(\phi^{+}(a)-\boldsymbol{\pi}^{+}(0, a)\right)\right)(\mathbf{I}-\mathbf{C})^{-1}, &
\end{array}
$$




$$
\begin{aligned}
& \boldsymbol{\pi}(0, r)=\Omega^{-1}\left(\phi^{+}(r)-\boldsymbol{\pi}^{+}(0, r)\right)(\mathbf{I}-\mathbf{C})^{-1}, \\
& \boldsymbol{\pi}(n, r)=\left(\boldsymbol{\pi}(n-1, r) \mathbf{D}-\Omega^{-1} \boldsymbol{\pi}^{+}(n, r)\right)(\mathbf{I}-\mathbf{C})^{-1}, \\
& a+1 \leq r \leq b, \\
& \boldsymbol{\pi}(n, b)=\left(\boldsymbol{\pi}(n-1, b) \mathbf{D}+\Omega^{-1}\left(\phi^{+}(n+b)-\boldsymbol{\pi}^{+}(n, b)\right)\right)(\mathbf{I}-\mathbf{C})^{-1}, \quad n \geq 1 .
\end{aligned}
$$

Proof. From Lemma 3.2, we obtain (4.1). Now setting $z=1$ in (3.8)-(3.11), we get

$$
\begin{aligned}
\boldsymbol{\pi}(0, a)(\mathbf{I}-\mathbf{C})= & -\boldsymbol{\pi}(0, a, 1) \mathbf{C}+\mathbf{p}(a-1,0) \mathbf{D}+\sum_{r=a}^{b} \boldsymbol{\pi}(a, r, 1) \mathbf{C}+\sum_{r=a}^{b} \boldsymbol{\pi}(a-1, r, 1) \mathbf{D}, \\
\boldsymbol{\pi}(0, r)(\mathbf{I}-\mathbf{C})= & -\boldsymbol{\pi}(0, r, 1) \mathbf{C}+\sum_{i=a}^{b} \boldsymbol{\pi}(r, i, 1) \mathbf{C}+\sum_{i=a}^{b} \boldsymbol{\pi}(r-1, i, 1) \mathbf{D}, a+1 \leq r \leq b, \\
\boldsymbol{\pi}(n, r)(\mathbf{I}-\mathbf{C})= & -\boldsymbol{\pi}(n, r, 1) \mathbf{C}+(\boldsymbol{\pi}(n-1, r)-\boldsymbol{\pi}(n-1, r, 1)) \mathbf{D}, n \geq 1, a \leq r \leq b-1, \\
\boldsymbol{\pi}(n, b)(\mathbf{I}-\mathbf{C})= & -\boldsymbol{\pi}(n, b, 1) \mathbf{C}+(\boldsymbol{\pi}(n-1, b)-\boldsymbol{\pi}(n-1, b, 1)) \mathbf{D}+\sum_{r=a}^{b} \boldsymbol{\pi}(n+b, r, 1) \mathbf{C} \\
& +\sum_{r=a}^{b} \boldsymbol{\pi}(n+b-1, r, 1) \mathbf{D}, n \geq 1 .
\end{aligned}
$$

Multiplying (4.6)-(4.9) by $\Omega$, using Lemmas 3.1 and 3.2, and the definition of departure epoch, we get the required relation between arbitrary and departure epochs as given in (4.2)-(4.5).

\subsection{Queue length and server content distribution at pre-arrival epoch}

Let $\mathbf{p}^{-}(n, 0),(0 \leq n \leq a-1)$ be the $1 \times m$ vectors whose $i$ th component $p_{i}^{-}(n, 0)$ is the steady-state probability that an arrival finds $n$ customers in the queue with server idle and phase of the arrival process $i$. Similarly, let $\pi^{-}(n, r),(n \geq 0, a \leq r \leq b)$ be the $1 \times m$ vectors whose $i$ th component $\pi_{i}^{-}(n, r)$ is the steady-state probability that an arrival finds $n(\geq 0)$ customers in the queue with server busy having $r(a \leq r \leq b)$ customers in service and phase of the arrival process being $i$. It can be easily shown (see [22]) that the vectors $\mathbf{p}^{-}(n, 0)$ and $\boldsymbol{\pi}^{-}(n, r)$ are given by

$$
\begin{array}{ll}
\mathbf{p}^{-}(n, 0)=\frac{\mathbf{p}(n, 0) \mathbf{D}}{\lambda^{*}}, & 0 \leq n \leq a-1, \\
\boldsymbol{\pi}^{-}(n, r)=\frac{\boldsymbol{\pi}(n, r) \mathbf{D}}{\lambda^{*}}, & a \leq r \leq b, n \geq 0 .
\end{array}
$$

\subsection{Queue length and server content distribution at outside observer's epoch}

In LAS-DA, since an outside observer's observation epoch falls in a time interval after the potential departure of a batch and before a potential arrival, the probability vector $\pi^{\circ}(n, r),(a \leq r \leq b, n \geq 0)$ that an outside observer sees $n$ customers in the queue and $r$ with the server is the same as that of the arbitrary epoch $\boldsymbol{\pi}(n, r),(a \leq r \leq b, n \geq 0)$, i.e. $\boldsymbol{\pi}^{\circ}(n, r)=\boldsymbol{\pi}(n, r),(a \leq r \leq b, n \geq 0)$.

This completes the theoretical analysis of the model. In the next section, we present a step-wise procedure for computing the distribution of queue and server content at various epochs. One can observe from Section 4 that in order to obtain these distributions, first we have to find the distribution at departure epoch which is discussed in the next section.

\section{Computing process to obtain the Distributions AT VARIOUs EPochs}

In this section we present the step-wise computing procedure for evaluation of the distribution at departure epoch. In order to extract the probability distribution from (3.31), first we have to determine the unknown 
probability vectors $\left\{\phi^{+}(n)\right\}_{n=0}^{b-1}$. Thus in total we have to determine $m b$ unknowns i.e. $\left\{\phi_{i}^{+}(n)\right\}_{n=0}^{b-1}, 1 \leq i \leq m$. We obtain these unknowns from (3.28) using the roots method given in Chaudhry et al. [13], Gupta et al. [18] and Pradhan and Gupta [22]. For this, we first obtain the expressions for $\mathbf{A}_{r}^{*}(x), a \leq r \leq b$, by considering commonly used service-time distributions.

\subsection{Evaluation of $\mathrm{A}_{r}^{*}(x), a \leq r \leq b$}

In this section, we obtain the expression for $\mathbf{A}_{r}^{*}(x), a \leq r \leq b$ when service-time distribution follows: (i) discrete phase-type (DPH) distribution (ii) arbitrary distribution. These distributions cover almost all types of distributions that arise in many real life situations.

\subsubsection{Service-time follows DPH distribution}

Let service-time follow a DPH distribution with representation $\operatorname{DPH}_{r}\left(\boldsymbol{\beta}_{r}, \mathbf{T}_{r}\right),(a \leq r \leq b)$, where $\boldsymbol{\beta}_{r}$ and $\mathbf{T}_{r}$ are row vectors and matrices, respectively, of dimension $\nu$. We have $s_{r}(k)=\boldsymbol{\beta}_{r} \mathbf{T}_{r}^{k-1} \mathbf{T}_{r}^{0}$, where $\mathbf{T}_{r}^{0}=\left(\mathbf{I}_{\nu}-\mathbf{T}_{r}\right) \mathbf{e}$. Using (2.2), we obtain

$$
\begin{aligned}
\mathbf{A}_{r}^{*}(x) & =\sum_{k=1}^{\infty}[\mathbf{C}+\mathbf{D} x]^{k} \otimes s_{r}(k), \\
& =\sum_{k=1}^{\infty}\left[\mathbf{I}_{m}(\mathbf{C}+\mathbf{D} x)^{k-1}(\mathbf{C}+\mathbf{D} x)\right] \otimes\left(\boldsymbol{\beta}_{r} \mathbf{T}_{r}^{k-1} \mathbf{T}_{r}^{0}\right) \\
& =\left(\mathbf{I}_{m} \otimes \boldsymbol{\beta}_{r}\right)\left(\sum_{k=1}^{\infty}(\mathbf{C}+\mathbf{D} x)^{k-1} \otimes \mathbf{T}_{r}^{k-1}\right)\left((\mathbf{C}+\mathbf{D} x) \otimes \mathbf{T}_{r}^{0}\right) \\
& =\left(\mathbf{I}_{m} \otimes \boldsymbol{\beta}_{r}\right)\left(\sum_{k=1}^{\infty}\left((\mathbf{C}+\mathbf{D} x) \otimes \mathbf{T}_{r}\right)^{k-1}\right)\left((\mathbf{C}+\mathbf{D} x) \otimes \mathbf{T}_{r}^{0}\right) \\
\mathbf{A}_{r}^{*}(x) & =\left(\mathbf{I}_{m} \otimes \boldsymbol{\beta}_{r}\right)\left(\mathbf{I}_{m \nu}-(\mathbf{C}+\mathbf{D} x) \otimes \mathbf{T}_{r}\right)^{-1}\left((\mathbf{C}+\mathbf{D} x) \otimes \mathbf{T}_{r}^{0}\right),
\end{aligned}
$$

where $\otimes$ is used for the Kronecker product.

Since the inverse term is appearing in the expression of $\mathbf{A}_{r}^{*}(x)$, we can write $\mathbf{A}_{r}^{*}(x)$ as

$$
\mathbf{A}_{r}^{*}(x)=\frac{\mathbf{X}_{r}(x)}{y_{r}(x)}
$$

where

$$
\mathbf{X}_{r}(x)=\left(\mathbf{I}_{m} \otimes \boldsymbol{\beta}_{r}\right) \operatorname{Adj}\left(\mathbf{I}_{m \nu}-(\mathbf{C}+\mathbf{D} x) \otimes \mathbf{T}_{r}\right)\left((\mathbf{C}+\mathbf{D} x) \otimes \mathbf{T}_{r}^{0}\right)
$$

and

$$
y_{r}(x)=\operatorname{Det}\left(\mathbf{I}_{m \nu}-(\mathbf{C}+\mathbf{D} x) \otimes \mathbf{T}_{r}\right) .
$$

Thus we can conclude that each element of the matrix $\mathbf{A}_{r}^{*}(x)$ is a rational function with the denominator as $y_{r}(x)$.

Remark 5.1. (i) If we set $\boldsymbol{\beta}_{r}=(1)$ and $\mathbf{T}_{r}=\left[1-\mu_{r}\right]$, we get $\mathbf{A}_{r}^{*}(x)$ for geometric service time distribution.

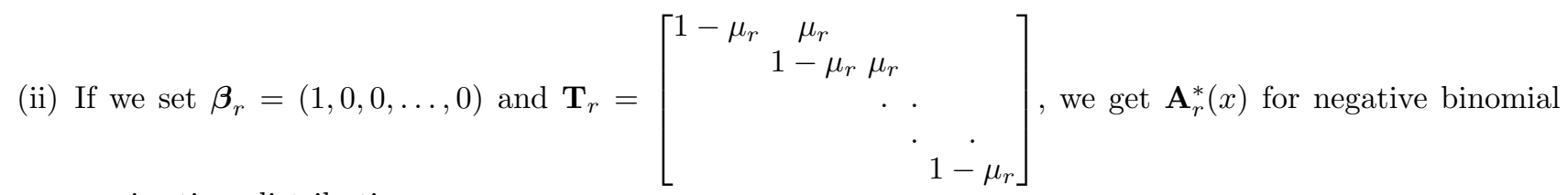
service time distribution. 


\subsubsection{Service-time follows arbitrary distribution}

Let service-time be arbitrarily distributed with maximum $K$ slots so that

$$
S_{r}^{*}(z)=\sum_{n=1}^{K} s_{r}(n) z^{n}, \quad \sum_{i=1}^{K} s_{r}(i)=1 .
$$

This leads to $\mathbf{A}_{r}^{*}(x)=\sum_{n=1}^{K} s_{r}(n)(\mathbf{C}+\mathbf{D} x)^{n}$.

Remark 5.2. If $s_{r}(K)=1$ and $s_{r}(i)=0$, (for all $i<K$ ) then we obtain $\mathbf{A}_{r}^{*}(x)$ for deterministic service time distribution with parameter $K$, i.e., $\mathbf{A}_{r}^{*}(x)=(\mathbf{C}+\mathbf{D} x)^{K}$.

Remark 5.3. From the above expressions of $\mathbf{A}_{r}^{*}(x)$, we can conclude that each element of $\mathbf{A}_{r}^{*}(x)$ can be written as $\mathbf{A}_{r}^{*}(x)=\frac{\mathbf{X}_{r}(x)}{y_{r}(x)}$. Note that for arbitrary service time distribution $y_{r}(x)=1$.

\subsection{Computing process for evaluation of distributions at departure epochs}

First we present step-wise computing process for the evaluation of unknown probability vector, then using it we extract the remaining probability vectors.

\subsubsection{Determination of unknown probability vectors}

As each element of the matrix $\mathbf{A}_{r}^{*}(x)$ is a rational function, we assume that the $(i, j)$-th element of $\mathbf{A}_{r}^{*}(x)$ is $\frac{X_{r ; i, j}(x)}{y_{r}(x)}$. So that the $(i, j)$-th element of $\left(x^{b} \mathbf{I}-\mathbf{A}_{b}^{*}(x)\right)$ is

$$
\left[x^{b} \mathbf{I}-\mathbf{A}_{b}^{*}(x)\right]_{i, j}=\frac{w_{i, j}(x)}{y_{b}(x)}, \text { where } w_{i, j}(x)= \begin{cases}x^{b} y_{b}(x)-X_{b ; i, j}(x), & i=j \\ -X_{b ; i, j}(x), & i \neq j\end{cases}
$$

Now (3.28) can be written in the form of $m$ simultaneous equations in $m$ unknowns $\phi_{j}^{+}(x), \quad 1 \leq j \leq m$.

$$
\begin{aligned}
w_{1,1}(x) \phi_{1}^{+}(x)+w_{2,1}(x) \phi_{2}^{+}(x)+\ldots+w_{m, 1}(x) \phi_{m}^{+}(x) & =\Theta_{1}(x) \\
w_{1,2}(x) \phi_{1}^{+}(x)+w_{2,2}(x) \phi_{2}^{+}(x)+\ldots+w_{m, 2}(x) \phi_{m}^{+}(x) & =\Theta_{2}(x) \\
\vdots & \vdots \\
w_{1, m}(x) \phi_{1}^{+}(x)+w_{2, m}(x) \phi_{2}^{+}(x)+\ldots+w_{m, m}(x) \phi_{m}^{+}(x)= & \Theta_{m}(x),
\end{aligned}
$$

where $\Theta_{j}(x), 1 \leq j \leq m$ is given as

$$
\begin{aligned}
\Theta_{j}(x)= & {\left[\prod _ { i = a + 1 } ^ { b - 1 } y _ { i } ( x ) \left\{\sum_{l=1}^{m} \sum_{n=0}^{a-1} \sum_{k=1}^{m} \phi_{k}^{+}(n)(\bar{D})_{k, l}^{(a-n)} X_{a ; l, j}(x) x^{b} y_{b}(x)\right.\right.} \\
& \left.-\sum_{l=1}^{m} \sum_{n=0}^{a-1} \phi_{l}^{+}(n) x^{n} X_{b ; l, j}(x) y_{a}(x)\right\}-\sum_{l=1}^{m} \sum_{n=a}^{b-1} \phi_{l}^{+}(n)\left\{x^{b} y_{b}(x) X_{n ; l, j}(x)\right. \\
& \left.\left.-x^{n} y_{n}(x) X_{b ; l, j}(x)\right\} \prod_{i=a, i \neq n}^{b-1} y_{i}(x)\right] /\left[\prod_{i=a}^{b-1} y_{i}(x)\right]
\end{aligned}
$$

Now solving the above system of equations using Cramer's rule, we obtain $\phi_{j}^{+}(x), 1 \leq j \leq m$, as

$$
\phi_{j}^{+}(x)=\frac{\left|V_{j}(x)\right|}{|V(x)|}, \quad 1 \leq j \leq m
$$


where both $V_{j}(x)$ and $V(x)$ are square matrices and their $(k, l)$-th element is given by

$$
\left[V_{j}(x)\right]_{k, l}=\left\{\begin{array}{ll}
v_{l, k}(x), & l \neq j \\
\Theta_{k}(x), & l=j
\end{array} \quad \text { and } \quad[V(x)]_{k, l}=v_{l, k}(x)\right.
$$

The $j$-th column of the square matrix $V_{j}(x)$ is replaced by $\left[\Theta_{1}(x), \Theta_{2}(x), \ldots, \Theta_{m}(x)\right]^{T}$ and all other elements are the same as those of $V(x)$.

Let us assume that $|V(x)|$ is a polynomial in $x$ which must possess a non-zero coefficient of power of $x$. Finally, we have

$$
\phi_{j}^{+}(x)=\frac{\Upsilon_{j}(x)}{\Upsilon(x)}, \quad 1 \leq j \leq m,
$$

where $\Upsilon_{j}(x)=\left|V_{j}(x)\right|$ and $\Upsilon(x)=|V(x)|$. To be more specific what we are having now is the pgf of only queue length distribution for each phase at a departure epoch. Till now we have not discussed about the determination of unknown probability vectors. To do this, we consider (5.5), and let us call $\Upsilon(x)=0$ as characteristic equation associated with the pgf of each phase. It can be easily shown that $\left|x^{b} \mathbf{I}-\mathbf{A}_{b}^{*}(x)\right| \equiv \frac{\Upsilon(x)}{\left\{y_{b}(x)\right\}^{m}}=0$ has exactly $m b$ roots inside and on the closed complex unit disk $|x| \leq 1$, see Gail et al. [16], p. 5. Let us assume that these roots are distinct and denote them as $x_{1}, x_{2}, \ldots, x_{m b}$ with $x_{m b}=1$. However, in case of repeated roots the procedure has to be modified slightly which is a standard procedure in the literature on queueing theory.

Analyticity of $\phi_{j}^{+}(x)$ in $|x| \leq 1$ implies that the roots $x_{1}, x_{2}, \ldots, x_{m b-1}$ of $\Upsilon(x)=0$ (the denominator of (5.5)) must coincide with that of numerator. Thus, by taking any one component of $\boldsymbol{\Phi}^{+}(x)$, say $\phi_{j}^{+}(x), \quad(1 \leq j \leq m)$ we are led to $m b-1$ equations as

$$
\Upsilon_{j}\left(x_{i}\right)=0, \quad 1 \leq i \leq m b-1
$$

The necessity of one more equation can be fulfilled by employing the normalizing condition $\boldsymbol{\Phi}^{+}(1) e=1$, which leads to

$$
\sum_{j=1}^{m} \Upsilon_{j}^{\prime}(1)=\Upsilon^{\prime}(1)
$$

Solving (5.6) and (5.7) together, we get the $m b$ unknowns $\phi_{j}^{+}(n), \quad(0 \leq n \leq b-1,1 \leq j \leq m)$.

\subsection{Extraction of probability vectors from bivariate VGF}

In the previous section, we have obtained unknown probability vectors $\left\{\boldsymbol{\phi}^{+}(n)\right\}_{n=0}^{b-1}$. So, we have bivariate $\operatorname{VGF} \tilde{\boldsymbol{\pi}}^{+}(x, \xi)$ (see, (3.31)) in completely known form. Now, our aim is to extract the probability vectors $\boldsymbol{\pi}^{+}(n, r), n \geq 0, a \leq r \leq b$. However, it is not easy to invert $\tilde{\boldsymbol{\pi}}^{+}(x, \xi)$ directly. To make it simpler we first collect the coefficient of $\xi^{j}, a \leq j \leq b$, from both the sides of (3.31), and they are given by

$$
\begin{aligned}
\text { coefficient of } \xi^{a}: \quad \sum_{n=0}^{\infty} \boldsymbol{\pi}^{+}(n, a) x^{n}= & \sum_{n=0}^{a} \boldsymbol{\phi}^{+}(n) \overline{\mathbf{D}}^{a-n} \mathbf{A}_{a}^{*}(x) . \\
\text { coefficient of } \xi^{j}: \quad \sum_{n=0}^{\infty} \boldsymbol{\pi}^{+}(n, j) x^{n}= & \boldsymbol{\phi}^{+}(j) \mathbf{A}_{j}^{*}(x), \quad a+1 \leq j \leq b-1 . \\
\text { coefficient of } \xi^{b}: \quad \sum_{n=0}^{\infty} \boldsymbol{\pi}^{+}(n, b) x^{n}= & {\left[\sum_{n=0}^{a-1} \boldsymbol{\phi}^{+}(n)\left\{\overline{\mathbf{D}}^{a-n} \mathbf{A}_{a}^{*}(x)-x^{n} \mathbf{I}\right\}\right.} \\
& \left.+\sum_{n=a}^{b-1} \boldsymbol{\phi}^{+}(n)\left\{\mathbf{A}_{n}^{*}(x)-x^{n} \mathbf{I}\right\}\right] \mathbf{A}_{b}^{*}(x)\left[x^{b} \mathbf{I}-\mathbf{A}_{b}^{*}(x)\right]^{-1} .
\end{aligned}
$$


Collecting the coefficient of $x^{n}$ from both the sides of (5.8) and (5.9) we get

$$
\begin{aligned}
\boldsymbol{\pi}^{+}(n, a) & =\sum_{n=0}^{a} \phi^{+}(n) \overline{\mathbf{D}}^{a-n} \mathbf{A}_{a}(n), & & n \geq 0 . \\
\boldsymbol{\pi}^{+}(n, j) & =\boldsymbol{\phi}^{+}(j) \mathbf{A}_{j}(n), & & a+1 \leq j \leq b-1, \quad n \geq 0 .
\end{aligned}
$$

Now, only vector $\boldsymbol{\pi}^{+}(n, b)$ is left to be determined. That can be done by inverting (5.10), where each component of the vector is a polynomial in $x$ for a specific service time distribution.

Let us denote $\sum_{n=0}^{\infty} \boldsymbol{\pi}^{+}(n, b) x^{n}$ as $\boldsymbol{\psi}^{+}(x)=\left[\psi_{1}^{+}(x), \psi_{2}^{+}(x), \ldots, \psi_{m}^{+}(x)\right]$ to make the analysis easier for the rest of the portion of this section. In order to extract the probability vectors from $\boldsymbol{\psi}^{+}(x)$, the same analysis as used in the previous section for $\mathbf{\Phi}^{+}(x)$ has to be carried out. In view of this, $\mathbf{\Phi}^{+}(x)$ and $\Theta_{j}(x)$ (used in the earlier case in (5.2)) has to be replaced by $\psi^{+}(x)$ and $F_{j}(x)$, respectively, where $F_{j}(x),(1 \leq j \leq m)$ is given by

$$
\begin{aligned}
F_{j}(x)= & {\left[\sum _ { i = 1 } ^ { m } \left\{\sum_{l=1}^{m}\left(\sum_{n=0}^{a-1} \sum_{k=1}^{m} \phi_{k}^{+}(n)(\bar{D})_{k, l}^{(a-n)} \prod_{v=a+1}^{b-1} y_{v}(x) X_{a ; l, i}(x)+\sum_{n=a}^{b-1} \phi_{l}^{+}(n) \prod_{v=a ; v \neq n}^{b-1} y_{v}(x) X_{n ; l, i}(x)\right)\right.\right.} \\
& \left.\left.-\left(\sum_{n=0}^{b-1} \phi_{i}^{+}(n) x^{n}\right) \prod_{v=a}^{b-1} y_{v}(x)\right\} X_{b ; i, j}(x)\right] /\left[\prod_{v=a}^{b-1} y_{v}(x)\right] .
\end{aligned}
$$

Therefore the simplified form of $\psi_{j}^{+}(x)$ is given by

$$
\psi_{j}^{+}(x)=\frac{\left|V_{j}(x)\right|}{|V(x)|}, \quad 1 \leq j \leq m,
$$

where both $V_{j}(x)$ and $V(x)$ represent square matrices with $(k, l)$-th elements given by

$$
\left[V_{j}(x)\right]_{k, l}=\left\{\begin{array}{ll}
v_{l, k}(x), & l \neq j \\
F_{k}(x), & l=j
\end{array} \quad \text { and } \quad[V(x)]_{k, l}=v_{l, k}(x)\right.
$$

The $j$-th column of the square matrix $V_{j}(x)$ is replaced by $\left[F_{1}(x), F_{2}(x), \ldots, F_{m}(x)\right]^{T}$ and all other elements are the same as those of $V(x)$.

Let us assume that $|V(x)|$ is a polynomial in $x$ which must possess a non-zero coefficient of power of $x$. Finally, we have

$$
\psi_{j}^{+}(x)=\frac{\Upsilon_{j}(x)}{\Upsilon(x)}, \quad 1 \leq j \leq m,
$$

where $\Upsilon_{j}(x)=\left|V_{j}(x)\right|$ and $\Upsilon(x)=|V(x)|$. As $\psi_{j}^{+}(x)$ is a rational function in completely known polynomials, we can proceed to find its partial fraction. Let us assume that $\Upsilon_{j}(x)$ and $\Upsilon(x)$ are the polynomials of degree $L_{1}$ and $M_{1}$, respectively.

We already know that $\Upsilon(x)=0$ has $m b$ roots inside or on the unit circle. So, there are total $\left(M_{1}-m b\right)$ distinct roots of $\Upsilon(x)=0$ in $|x|>1$ (for repeated roots see Rem. 5.4). Let us denote these roots by $\alpha_{1}, \alpha_{2}, \ldots, \alpha_{M_{1}-m b}$. Now based on the value of $L_{1}$ and $M_{1}$, the following two cases arise:

Case 1. $L_{1} \geq M_{1}$.

Applying the partial-fraction expansion, the rational function $\psi_{j}^{+}(x)(1 \leq j \leq m)$, can be uniquely written as

$$
\psi_{j}^{+}(x)=\sum_{i=0}^{L_{1}-M_{1}} \tau_{i, j} x^{i}+\sum_{k=1}^{M_{1}-m b} \frac{\gamma_{k, j}}{\alpha_{k}-x}
$$


for some constants $\tau_{i, j}$ and $\gamma_{k, j}$ 's. The first sum is the result of the division of the polynomial $\Upsilon_{j}(x)$ by $\Upsilon(x)$ and the constants $\tau_{i, j}$ are the coefficients of the resulting quotient. Using the residue theorem, we have

$$
\gamma_{k, j}=-\frac{\Upsilon_{j}\left(\alpha_{k}\right)}{\Upsilon^{\prime}\left(\alpha_{k}\right)}, \quad k=1,2, \ldots, M_{1}-m b
$$

Now, collecting the coefficient of $x^{n}$ from both the sides of (5.17), we get

$$
\pi_{j}^{+}(n, b)=\tau_{n, j}+\sum_{k=1}^{M_{1}-m b} \frac{\gamma_{k, j}}{\alpha_{k}^{n+1}}, \quad n \geq 0 .
$$

Case 2. $L_{1}<M_{1}$.

Using partial-fraction technique on $\psi_{j}^{+}(x)$ we have

$$
\psi_{j}^{+}(x)=\sum_{k=1}^{M_{1}-m b} \frac{\gamma_{k, j}}{\alpha_{k}-x},
$$

where

$$
\gamma_{k, j}=-\frac{\Upsilon_{j}\left(\alpha_{k}\right)}{\Upsilon^{\prime}\left(\alpha_{k}\right)}, \quad k=1,2, \ldots, M_{1}-m b
$$

Now, collecting the coefficient of $x^{n}$ from both the sides of (5.19), we obtain

$$
\pi_{j}^{+}(n, b)=\sum_{k=1}^{M_{1}-m b} \frac{\gamma_{k, j}}{\alpha_{k}^{n+1}}, \quad n \geq 0 .
$$

This completes the analysis of obtaining the departure epoch probability vectors presented in (5.11), (5.12), (5.18) and (5.20).

Remark 5.4. In this paper we are assuming that all the roots of $\Upsilon(x)=0$ are distinct, for repeated roots slight modification is needed. For that, one may refer to Pradhan and Gupta [22,23].

\section{Special CASES}

In this section we discuss some special cases of the model.

\subsection{D-MAP $/ G / 1 / \infty$ queue}

We assume that the server provides individual service to the customers, according to the order of their arrival, i.e., $a=1, b=1$. As a result, the question of dependency of service rate on the batch size does not arise, hence $G_{n}=G$. Therefore, our model reduces to the D-MAP $/ G / 1 / \infty$ queue.

From (3.28) we get the VGF of the queue content at a departure epoch as

$$
\boldsymbol{\Phi}^{+}(x)\left(x \mathbf{I}-\mathbf{A}_{1}^{*}(x)\right)=\phi^{+}(0)\left((\overline{\mathbf{D}}) x \mathbf{A}_{1}^{*}(x)-\mathbf{A}_{1}^{*}(x)\right) .
$$

The probability vectors of the queue content at arbitrary and pre-arrival epochs are given by

$$
\begin{array}{ll}
\mathbf{p}(0,0)=\Omega^{-1} \boldsymbol{\phi}^{+}(0)(\mathbf{I}-\mathbf{C})^{-1} \\
\boldsymbol{\pi}(0,1)=\left(\mathbf{p}(0,0) \mathbf{D}+\Omega^{-1}\left(\boldsymbol{\phi}^{+}(1)-\boldsymbol{\pi}^{+}(0,1)\right)\right)(\mathbf{I}-\mathbf{C})^{-1}, \\
\boldsymbol{\pi}(n, 1)=\left(\boldsymbol{\pi}(n-1,1) \mathbf{D}+\Omega^{-1}\left(\boldsymbol{\phi}^{+}(n+1)-\boldsymbol{\pi}^{+}(n, 1)\right)\right)(\mathbf{I}-\mathbf{C})^{-1}, & n \geq 1 .
\end{array}
$$




$$
\begin{aligned}
& \mathbf{p}^{-}(0,0)=\frac{\mathbf{p}(0,0) \mathbf{D}}{\lambda^{*}} \\
& \boldsymbol{\pi}^{-}(n, 1)=\frac{\boldsymbol{\pi}(n, 1) \mathbf{D}}{\lambda^{*}}
\end{aligned}
$$

Here $\boldsymbol{\pi}^{+}(n, 1)=\phi^{+}(n), n \geq 0$. One may note that the waiting-time analysis of D-MAP/G/1 queue can be obtained from those of Samanta [24] by considering $D_{n}=0, n \geq 2$.

\subsection{D-MAP $/ G^{b} / 1 / \infty$ queue}

We assume that the server provides service to the customers in batches of fixed size say b, i.e., $a=b$. Moreover, the service rate does not depend on the service batch size, i.e., $G_{n}=G$. Thus, our model reduces to D-MAP $/ G^{b} / 1 / \infty$ queue.

From (3.28) we get the VGF of the queue content at a departure epoch as

$$
\mathbf{\Phi}^{+}(x)\left(x^{b} \mathbf{I}-\mathbf{A}^{*}(x)\right)=\sum_{n=a}^{b-1} \boldsymbol{\phi}^{+}(n)\left(x^{b} \mathbf{A}^{*}(x)-x^{n} \mathbf{A}^{*}(x)\right) .
$$

The probability vectors of the queue content at arbitrary and pre-arrival epochs are given by

$$
\begin{aligned}
\mathbf{p}(n, 0) & =\Omega^{-1} \sum_{j=0}^{n} \phi^{+}(j)(\overline{\mathbf{D}})^{(n-j)}(\mathbf{I}-\mathbf{C})^{-1}, & & 0 \leq n \leq b-1 . \\
\boldsymbol{\pi}(0, b) & =\left(\mathbf{p}(b-1,0) \mathbf{D}+\Omega^{-1}\left(\boldsymbol{\phi}^{+}(b)-\boldsymbol{\pi}^{+}(0, b)\right)\right)(\mathbf{I}-\mathbf{C})^{-1}, & & \\
\boldsymbol{\pi}(n, b) & =\left(\boldsymbol{\pi}(n-1, b) \mathbf{D}+\Omega^{-1}\left(\phi^{+}(n+b)-\boldsymbol{\pi}^{+}(n, b)\right)\right)(\mathbf{I}-\mathbf{C})^{-1}, & & n \geq 1 . \\
\mathbf{p}^{-}(n, 0) & =\frac{\mathbf{p}(n, 0) \mathbf{D}}{\lambda^{*}}, & & 0 \leq n \leq b-1, \\
\boldsymbol{\pi}^{-}(n, b) & =\frac{\boldsymbol{\pi}(n, b) \mathbf{D}}{\lambda^{*}}, & & n \geq 0 .
\end{aligned}
$$

Remark 6.1. In the case of $\mathrm{D}-\mathrm{MAP} / G / 1 / \infty$ and $\mathrm{D}-\mathrm{MAP} / G^{b} / 1 / \infty$ queues, only the VGF of the queue length can be obtained since in both the cases the server serves only a fixed number of customers, i.e., one and $b$, respectively.

\subsection{D-MAP $/ G^{(a, b)} / 1 / \infty$ queue}

The results of this model can be obtained by dropping the batch-size dependency service in our model, i.e., we assume $G_{n}=G$. From (3.28) we get the VGF of the queue content at a departure epoch as

$$
\begin{aligned}
\mathbf{\Phi}^{+}(x)\left(x^{b} \mathbf{I}-\mathbf{A}^{*}(x)\right)= & \sum_{n=0}^{a-1} \boldsymbol{\phi}^{+}(n)\left((\overline{\mathbf{D}})^{(a-n)} x^{b} \mathbf{A}^{*}(x)-x^{n} \mathbf{A}^{*}(x)\right) \\
& +\sum_{n=a}^{b-1} \boldsymbol{\phi}^{+}(n)\left(x^{b} \mathbf{A}^{*}(x)-x^{n} \mathbf{A}^{*}(x)\right) .
\end{aligned}
$$

Joint VGF of the queue and server content distribution at a departure epoch is given by

$$
\tilde{\boldsymbol{\pi}}^{+}(x, \xi)=\left(\sum_{n=0}^{a-1} \boldsymbol{\phi}^{+}(n)\left(\left(\xi^{b}-\xi^{a}\right)(\overline{\mathbf{D}})^{(a-n)} \mathbf{A}^{*}(x) \mathbf{A}^{*}(x)+(\overline{\mathbf{D}})^{(a-n)} \xi^{a} x^{b} \mathbf{A}^{*}(x)-\xi^{b} x^{n} \mathbf{A}^{*}(x)\right)\right.
$$




$$
\left.+\sum_{n=a}^{b-1} \boldsymbol{\phi}^{+}(n)\left(\left(\xi^{b}-\xi^{n}\right) \mathbf{A}^{*}(x) \mathbf{A}^{*}(x)+\xi^{n} x^{b} \mathbf{A}^{*}(x)-\xi^{b} x^{n} \mathbf{A}^{*}(x)\right)\right)\left(x^{b} \mathbf{I}-\mathbf{A}^{*}(x)\right)^{-1} .
$$

Joint queue and server content distribution at arbitrary and pre-arrival epochs are the same as given in (4.1)(4.5) and (4.10), (4.11), respectively.

\section{Performance measures}

Having found the probability vectors $\mathbf{p}(n, 0),(0 \leq n \leq a-1), \boldsymbol{\pi}(n, r),(a \leq r \leq b, n \geq 0)$, the other significant distributions of interest can be easily obtained and are given below:

- Distribution of the number of customers in the system at an arbitrary epoch (including number of customers with the server) is given by

$$
p_{n}^{\text {system }}= \begin{cases}\mathbf{p}(n, 0) \boldsymbol{e} & 0 \leq n \leq a-1, \\ \sum_{r=a}^{\min (b, n)} \boldsymbol{\pi}(n-r, r) \boldsymbol{e} & a \leq n \leq b, \\ \sum_{r=a}^{b} \boldsymbol{\pi}(n-r, r) \boldsymbol{e} & n \geq b+1 .\end{cases}
$$

- Distribution of the number of customers in the queue at arbitrary epoch is given by

$$
p_{n}^{\text {queue }}= \begin{cases}\mathbf{p}(n, 0) \boldsymbol{e}+\sum_{r=a}^{b} \boldsymbol{\pi}(n, r) \boldsymbol{e} & 0 \leq n \leq a-1, \\ \sum_{r=a}^{b} \boldsymbol{\pi}(n, r) \boldsymbol{e} & n \geq a .\end{cases}
$$

- Distribution of the number of customers in service given that server is busy

$$
p_{r}^{\text {server }}=c \sum_{n=0}^{\infty} \boldsymbol{\pi}(n, r) \boldsymbol{e}, \quad a \leq r \leq b,
$$

where $c^{-1}=\left[1-\sum_{n=0}^{a-1} \mathbf{p}(n, 0) \boldsymbol{e}\right]=P_{\text {busy }}$.

It is very much essential to study the performance measures of the queueing system as they play a notable role in designing and improving the efficiency of the system. Some performance measures are listed below:

- Average number of customers waiting in the queue $\left(L_{q}\right)=\sum_{n=0}^{\infty} n p_{n}^{\text {queue }}$.

- Average number of customers in the system $(L)=\sum_{n=0}^{\infty} n p_{n}^{\text {system }}$.

- Average number of customers with the server $\left(L_{s}\right)=\sum_{r=a}^{b} r p_{r}^{\text {server }}$.

- Average waiting time of a customer in the queue $\left(W_{q}\right)=\frac{L_{q}}{\lambda^{*}}$, as well as in the system $(W)=\frac{L}{\lambda^{*}}$.

- The probability that the server is idle $\left(P_{\text {idle }}\right)=\sum_{n=0}^{a-1} \mathbf{p}(n, 0) \boldsymbol{e}$.

Besides these performance measures a system designer may be interested in knowing information about the filling degree which is defined as the ratio of average number of customers in a served batch to the maximum number of customers that can be served, i.e., $\frac{L_{s}}{b}$ by which server's efficiency can be upgraded.

\section{NUMERICAL EXAMPLES}

In this section, we illustrate the methodology and the results derived in previous sections through some numerical examples which have been done using Maple 15 on PC having configuration Intel (R) Core (TM) i5-3470 CPU Processor @ 3.20 GHz with 4.00 GB of RAM. Though several results have been generated, a few of 
TABLE 1. DPH distribution for different batch-size.

\begin{tabular}{|c|c|c|c|c|c|c|c|c|c|}
\hline Batch size & $\boldsymbol{\beta}_{n}$ & & & & $\mathbf{T}_{n}$ & & & & $S_{n}$ \\
\hline 6 & $(0.3$ & 0.1 & 0.2 & & $\left(\begin{array}{l}0.6 \\
0.0 \\
0.0 \\
0.1\end{array}\right.$ & $\begin{array}{l}0.0 \\
0.6 \\
0.0 \\
0.1\end{array}$ & $\begin{array}{l}0.0 \\
0.1 \\
0.5 \\
0.0\end{array}$ & $\left.\begin{array}{l}0.3 \\
0.0 \\
0.2 \\
0.7\end{array}\right)$ & 6.604651 \\
\hline 7 & $(0.4$ & 0.2 & $0.4)$ & & $\left(\begin{array}{l}0.8 \\
0.1 \\
0.0\end{array}\right.$ & $\begin{array}{l}0.0 \\
0.6 \\
0.1\end{array}$ & $\left.\begin{array}{l}0.1 \\
0.1 \\
0.8\end{array}\right)$ & & 8.461538 \\
\hline 8 & $(0.25$ & 0.25 & 0.25 & $0.25)$ & $\left(\begin{array}{l}0.4 \\
0.0 \\
0.0 \\
0.2\end{array}\right.$ & $\begin{array}{l}0.0 \\
0.7 \\
0.0 \\
0.1\end{array}$ & $\begin{array}{l}0.0 \\
0.2 \\
0.5 \\
0.0\end{array}$ & $\left.\begin{array}{l}0.5 \\
0.0 \\
0.3 \\
0.6\end{array}\right)$ & 8.678160 \\
\hline 9 & $(0.5$ & 0.3 & $0.2)$ & & $\left(\begin{array}{l}0.7 \\
0.1 \\
0.1\end{array}\right.$ & $\begin{array}{l}0.1 \\
0.7 \\
0.2\end{array}$ & $\left.\begin{array}{l}0.1 \\
0.1 \\
0.6\end{array}\right)$ & & 10.000000 \\
\hline 10 & $(0.1$ & 0.1 & $0.8)$ & & $\left(\begin{array}{l}0.7 \\
0.2 \\
0.1\end{array}\right.$ & $\begin{array}{l}0.2 \\
0.6 \\
0.0 \\
\end{array}$ & $\left.\begin{array}{l}0.1 \\
0.1 \\
0.8\end{array}\right)$ & & 14.500000 \\
\hline
\end{tabular}

them are presented here which may be useful to researchers and practitioners. Numerical results for two different service-time distributions viz. discrete phase-type and negative binomial are given in the following examples. All the results are presented in 6 decimal places for sake of brevity. While dealing with the communication channel, one may encounter transmission errors which may arise due to fading channel, electrical defectiveness, unfavorable weather, etc. Discrete phase-type distribution (DPH) is a powerful tool to regulate this error. It also covers a wide range of almost all relevant discrete distributions such as geometric and negative binomial. Moreover, negative binomial distribution is one of the significant discrete distribution which can capture several real-life scenarios. Keeping this in mind, discrete phase type and negative binomial distributions have been considered as service time distributions in two examples. The purpose of these two examples is to show the applicability and simple usability of the proposed procedure and results.

Example 8.1. D-MAP/DPH $\mathrm{DH}_{n}^{(6,10)} / 1$ queue. Here the service time distribution is discrete phase (DPH)-type.

In this example the D-MAP is represented by the matrices

$$
\mathbf{C}=\left(\begin{array}{lll}
0.30 & 0.10 & 0.15 \\
0.35 & 0.05 & 0.20 \\
0.15 & 0.10 & 0.15
\end{array}\right) \quad \text { and } \quad \mathbf{D}=\left(\begin{array}{ccc}
0.10 & 0.25 & 0.10 \\
0.20 & 0.15 & 0.05 \\
0.45 & 0.05 & 0.10
\end{array}\right)
$$

that gives $\overline{\boldsymbol{\pi}}=[0.489130,0.260869,0.2500000]$ and $\lambda^{*}=0.474456$. The DPH-type distribution has the representation $(\boldsymbol{\beta}, \mathbf{T})$, where $\boldsymbol{\beta}$ is a row vector of order $\nu$ and $\mathbf{T}$ is a square matrix of order $\nu$. Other parameters are: $a=6, b=10$, and the batch-size dependent service time distribution for $\operatorname{DPH}_{n}\left(\boldsymbol{\beta}_{n}, \mathbf{T}_{n}\right), 6 \leq n \leq 10$ is given in the Table 1.

So $\rho=0.687961$. The joint queue and server content distribution for D-MAP $/ \mathrm{DPH}_{n}^{(6,10)} / 1$ queue, at different epochs has been displayed in Tables 2 and 3. 
TABLE 2. Joint distribution of queue and server content and phase of the arrival process at departure epoch for D-MAP $/ G_{n}^{(6,10)} / 1$ queue with $G \sim \mathrm{PH}$.

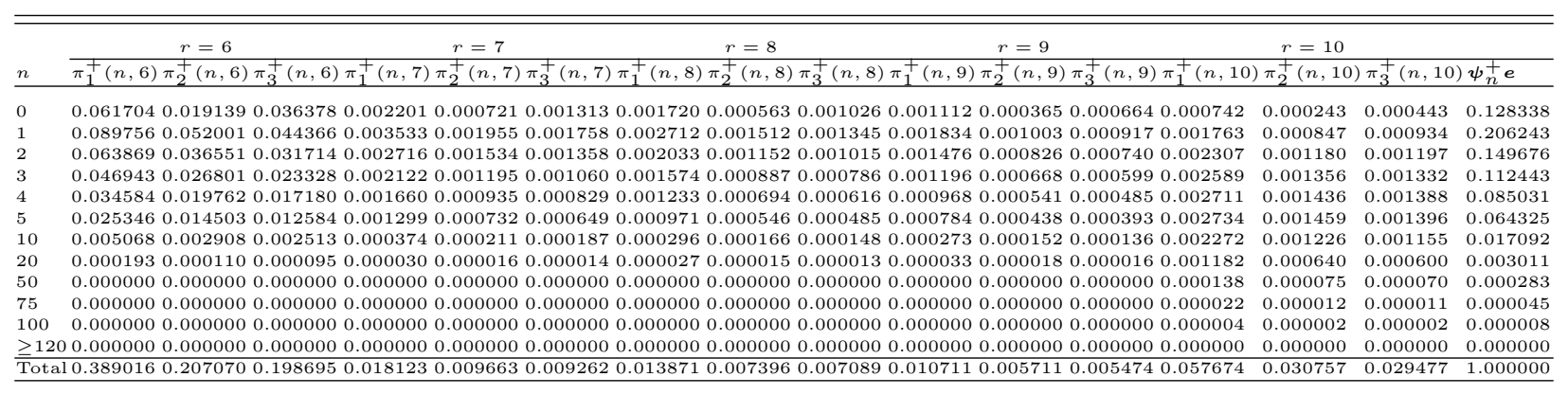

Example 8.2. D-MAP $/ \mathrm{NB}_{n}^{(4,7)} / 1$ queue. In this case the service time distribution is negative binomial (NB).

The matrices corresponding to the D-MAP are given by

$$
\mathbf{C}=\left(\begin{array}{ccc}
0.4 & 0.1 & 0.05 \\
0.25 & 0.05 & 0.30 \\
0.10 & 0.15 & 0.15
\end{array}\right) \text { and } \mathbf{D}=\left(\begin{array}{ccc}
0.15 & 0.20 & 0.10 \\
0.15 & 0.20 & 0.05 \\
0.05 & 0.45 & 0.10
\end{array}\right)
$$

That gives $\overline{\boldsymbol{\pi}}=[0.398305,0.355932,0.245762]$ and $\lambda^{*}=0.469067$. Other input parameters are: $a=4, b=7$, and mean service times of NB distribution for batch size dependent service time distributions are taken as

\begin{tabular}{|c|c|}
\hline Batch size $(r)$ & $S_{r}$ \\
\hline 4 & 3.000000 \\
5 & 4.000000 \\
6 & 5.666667 \\
7 & 9.000000 \\
\hline
\end{tabular}

So $\rho=0.603087$. The joint queue and server content distribution for D-MAP/NB$n^{(4,7)} / 1$ queue, at different epochs has been displayed in Tables 4 and 5 .

Now, we investigate the effect of correlation of arrival process on a few performance measures viz, filling degree, average queue length, average waiting time in the system. For this, we choose arrival processes with negative, zero, and positive correlation. The input parameters are given below:

Positive correlation: we choose following set of matrices with correlation $=0.2806$.

$$
\mathbf{C}=\left(\begin{array}{ll}
0.9130 & 0.0090 \\
0.0060 & 0.5992
\end{array}\right), \quad \mathbf{D}=\left(\begin{array}{ll}
0.0770 & 0.0090 \\
0.0040 & 0.3908
\end{array}\right)
$$

Zero correlation: we choose following set of matrices with zero correlation.

$$
\mathbf{C}=\left(\begin{array}{ll}
0.4364 & 0.2636 \\
0.5364 & 0.1636
\end{array}\right), \quad \mathbf{D}=\left(\begin{array}{ll}
0.2 & 0.1 \\
0.1 & 0.2
\end{array}\right)
$$

Negative correlation: we choose following set of matrices with correlation $=-0.053120$.

$$
\mathbf{C}=\left(\begin{array}{ll}
0.2364 & 0.0 \\
0.5364 & 0.0636
\end{array}\right), \quad \mathbf{D}=\left(\begin{array}{ll}
0.5 & 0.2636 \\
0.00 & 0.4
\end{array}\right)
$$


TABLE 3. Joint distribution of queue and server content and phase of the arrival process at arbitrary epoch for D-MAP $/ G_{n}^{(5,9)} / 1$ queue with $G \sim \mathrm{PH}$.

\begin{tabular}{|c|c|c|c|c|c|c|c|c|c|c|c|c|}
\hline \multirow[b]{2}{*}{$n$} & \multicolumn{3}{|c|}{$r=0$} & \multicolumn{3}{|c|}{$r=6$} & \multicolumn{3}{|c|}{$r=7$} & \multicolumn{3}{|c|}{$r=8$} \\
\hline & $p_{1}(n, 0)$ & $p_{2}(n, 0)$ & $p_{3}(n, 0)$ & $\pi_{1}(n, 6)$ & $\pi_{2}(n, 6)$ & $\pi_{3}(n, 6)$ & $\pi_{1}(n, 7)$ & $\pi_{2}(n, 7)$ & $\pi_{3}(n, 7)$ & $\pi_{1}(n, 8)$ & $\pi_{2}(n, 8)$ & $\pi_{3}(n, 8)$ \\
\hline 0 & 0.009760 & 0.003225 & 0.005833 & 0.049774 & 0.027979 & 0.024815 & 0.002449 & 0.001110 & 0.001335 & 0.001869 & 0.000847 & 0.001019 \\
\hline 1 & 0.024974 & 0.011735 & 0.013344 & 0.036004 & 0.020578 & 0.017882 & 0.001886 & 0.001040 & 0.000940 & 0.001437 & 0.000792 & 0.000716 \\
\hline 2 & 0.035927 & 0.017995 & 0.018805 & 0.026319 & 0.015058 & 0.013068 & 0.001463 & 0.000825 & 0.000731 & 0.001120 & 0.000631 & 0.000560 \\
\hline 3 & 0.044173 & 0.022675 & 0.022910 & 0.019207 & 0.010998 & 0.009533 & 0.001143 & 0.000644 & 0.000570 & 0.000882 & 0.000496 & 0.000441 \\
\hline 4 & 0.050406 & 0.026215 & 0.026015 & 0.013966 & 0.008004 & 0.006930 & 0.000891 & 0.000502 & 0.000445 & 0.000695 & 0.000391 & 0.000347 \\
\hline 5 & 0.055122 & 0.028893 & 0.028364 & 0.010125 & 0.005806 & 0.005023 & 0.000694 & 0.000391 & 0.000347 & 0.000548 & 0.000308 & 0.000274 \\
\hline 10 & & & & 0.001989 & 0.001141 & 0.000986 & 0.000197 & 0.000111 & 0.000098 & 0.000167 & 0.000094 & 0.000084 \\
\hline 20 & & & & 0.000075 & 0.000043 & 0.000037 & 0.000015 & 0.000009 & 0.000008 & 0.000015 & 0.000009 & 0.000008 \\
\hline 50 & & & & 0.000000 & 0.000000 & 0.000000 & 0.000000 & 0.000000 & 0.000000 & 0.000000 & 0.000000 & 0.000000 \\
\hline 75 & & & & 0.000000 & 0.000000 & 0.000000 & 0.000000 & 0.000000 & 0.000000 & 0.000000 & 0.000000 & 0.000000 \\
\hline 100 & & & & 0.000000 & 0.000000 & 0.000000 & 0.000000 & 0.000000 & 0.000000 & 0.000000 & 0.000000 & 0.000000 \\
\hline$\geq 120$ & & & & 0.000000 & 0.000000 & 0.000000 & 0.000000 & 0.000000 & 0.000000 & 0.000000 & 0.000000 & 0.000000 \\
\hline Total & 0.220364 & $\begin{array}{c}0.110740 \\
r=9\end{array}$ & 0.115273 & 0.181736 & $\begin{array}{c}0.103536 \\
r=10\end{array}$ & 0.090314 & 0.010959 & 0.005886 & 0.005584 & 0.008603 & 0.004619 & 0.004384 \\
\hline$n$ & $\pi_{1}(n, 9)$ & $\pi_{2}(n, 9)$ & $\pi_{3}(n, 9)$ & $\pi_{1}(n, 10)$ & $\pi_{2}(n, 10)$ & $\pi_{3}(n, 10)$ & & & & & & \\
\hline 0 & 0.001475 & 0.000663 & 0.000806 & 0.001170 & 0.000523 & 0.000641 & 0.135302 & & & & & \\
\hline 1 & 0.001181 & 0.000646 & 0.000590 & 0.001904 & 0.000943 & 0.000999 & 0.137597 & & & & & \\
\hline 2 & 0.000950 & 0.000532 & 0.000477 & 0.002350 & 0.001210 & 0.001217 & 0.139246 & & & & & \\
\hline 3 & 0.000770 & 0.000430 & 0.000386 & 0.002606 & 0.001366 & 0.001340 & 0.140577 & & & & & \\
\hline 4 & 0.000623 & 0.000348 & 0.000312 & 0.002730 & 0.001446 & 0.001399 & 0.141673 & & & & & \\
\hline 5 & 0.000505 & 0.000282 & 0.000253 & 0.002763 & 0.001473 & 0.001412 & 0.142591 & & & & & \\
\hline 10 & 0.000175 & 0.000098 & 0.000088 & 0.002337 & 0.001261 & 0.001188 & 0.010022 & & & & & \\
\hline 20 & 0.000021 & 0.000011 & 0.000010 & 0.001227 & 0.000664 & 0.000623 & 0.002781 & & & & & \\
\hline 50 & 0.000000 & 0.000000 & 0.000000 & 0.000143 & 0.000077 & 0.000073 & 0.0002948 & & & & & \\
\hline 75 & 0.000000 & 0.000000 & 0.000000 & 0.000023 & 0.000013 & 0.000012 & 0.000048 & & & & & \\
\hline 100 & 0.000000 & 0.000000 & 0.000000 & 0.000004 & 0.000002 & 0.000002 & 0.000008 & & & & & \\
\hline$\geq 120$ & 0.000000 & 0.000000 & 0.000000 & 0.000000 & 0.000000 & 0.000000 & 0.000000 & & & & & \\
\hline \multirow[t]{2}{*}{ Total } & 0.007656 & 0.004106 & 0.003904 & 0.059808 & 0.031978 & 0.030537 & 1.000000 & & & & & \\
\hline & & -0.11 & $\begin{array}{lc}1, & L_{q} \\
78, & W \\
\end{array}$ & $\begin{array}{l}=4.617451, \\
=17.98291\end{array}$ & $\begin{array}{r}L_{s}=7 . \\
5, \quad W_{q}=\end{array}$ & $\begin{array}{l}072806 \\
9.732085 \\
\end{array}$ & & & & & & \\
\hline
\end{tabular}

TABLE 4. Joint distribution of queue and server content and phase of the arrival process at departure epoch for D-MAP $/ G_{n}^{(4,7)} / 1$ queue, with $G \sim \mathrm{NB}$.

\begin{tabular}{|c|c|c|c|c|c|c|c|c|c|c|c|c|c|}
\hline \multirow[b]{2}{*}{$n$} & \multicolumn{3}{|c|}{$r=4$} & \multicolumn{3}{|c|}{$r=5$} & \multicolumn{3}{|c|}{$r=6$} & \multicolumn{3}{|c|}{$r=7$} & \multirow[b]{2}{*}{$\boldsymbol{\psi}_{n}^{+} \boldsymbol{e}$} \\
\hline & $\pi_{1}^{+}(n, 4)$ & $\pi_{2}^{+}(n, 4)$ & $\pi_{3}^{+}(n, 4)$ & $\pi_{1}^{+}(n, 5)$ & $\pi_{2}^{+}(n, 5)$ & $\pi_{3}^{+}(n, 5)$ & $\pi_{1}^{+}(n, 6)$ & $\pi_{2}^{+}(n, 6)$ & $\pi_{3}^{+}(n, 6)$ & $\pi_{1}^{+}(n, 7)$ & $\pi_{2}^{+}(n, 7)$ & $\pi_{3}^{+}(n, 7)$ & \\
\hline 0 & 0.121956 & 0.042343 & 0.076275 & 0.001589 & 0.000563 & 0.000865 & 0.000425 & 0.000151 & 0.000224 & 0.000094 & 0.000033 & 0.000047 & 0.244565 \\
\hline 1 & 0.134098 & 0.144599 & 0.087626 & 0.002001 & 0.001966 & 0.001316 & 0.000626 & 0.000557 & 0.000402 & 0.000204 & 0.000146 & 0.000122 & 0.373663 \\
\hline 2 & 0.073266 & 0.086478 & 0.047233 & 0.001404 & 0.001522 & 0.000904 & 0.000546 & 0.000541 & 0.000347 & 0.000268 & 0.000224 & 0.000165 & 0.212898 \\
\hline 3 & 0.032838 & 0.040127 & 0.021078 & 0.000813 & 0.000914 & 0.000521 & 0.000398 & 0.000411 & 0.000253 & 0.000285 & 0.000254 & 0.000177 & 0.098069 \\
\hline 4 & 0.013417 & 0.016701 & 0.008592 & 0.000430 & 0.000493 & 0.000275 & 0.000266 & 0.000281 & 0.000169 & 0.000271 & 0.000250 & 0.000169 & 0.041314 \\
\hline 5 & 0.005194 & 0.006540 & 0.003321 & 0.000215 & 0.000251 & 0.000138 & 0.000169 & 0.000181 & 0.000108 & 0.000241 & 0.000227 & 0.000150 & 0.016735 \\
\hline 10 & 0.000031 & 0.000040 & 0.000019 & 0.000004 & 0.000005 & 0.000003 & 0.000012 & 0.00 & 0.000007 & 0.000087 & 0.000085 & 0.000054 & 0.000360 \\
\hline 15 & 0.000000 & 0.000000 & 0.000000 & 0.000000 & 0.000000 & 0.000000 & 0.000000 & 0.000000 & 0.000000 & 0.000024 & 0.000024 & 0.000015 & 0.000063 \\
\hline 20 & 0.000000 & 0.000000 & 0.000000 & 0.000000 & 0.000000 & 0.000000 & 0.000000 & 0.000000 & 0.000000 & 0.000006 & 0.000006 & 0.000004 & 0.000016 \\
\hline$\geq 30$ & 0.000000 & 0.000000 & 0.000000 & 0.000000 & 0.000000 & 0.000000 & 0.000000 & 0.000000 & 0.000000 & 0.000000 & 0.000000 & 0.000000 & 0.000000 \\
\hline Total & 0.383811 & 0.340657 & 0.246069 & 0.006651 & 0.005942 & 0.004147 & 0.002685 & 0.002400 & 0.001667 & 0.002374 & 0.002122 & 0.001468 & 1.000000 \\
\hline
\end{tabular}

Service time distribution is taken as DPH with $a=5, b=8$ and

$$
\boldsymbol{\beta}_{r}=(0.5,0.5), \mathbf{T}_{r}=\left(\begin{array}{ll}
1-\theta_{r} & \theta_{r} \\
0 & 1-\theta_{r}
\end{array}\right) .
$$

By varying the values of $\theta_{r}(5 \leq r \leq 8)$, we get different $\mu_{r}{ }^{-1}=S_{r}$ which eventually lead to different $\rho$. In Table 6 , we present how the different values of $\mu_{r}{ }^{-1}$ lead to different values of $\rho$.

In Figure 2, we study the effect of correlation (positive, zero and negative) on the filling degree with respect to the traffic intensity $\rho$. It may be observed that for a very low value of $\rho$, the filling degree is the same for positive 
TABLE 5. Joint distribution of queue and server content and phase of the arrival process at arbitrary epoch for D-MAP $/ G_{n}^{(4,7)} / 1$ queue, with $G \sim \mathrm{NB}$.

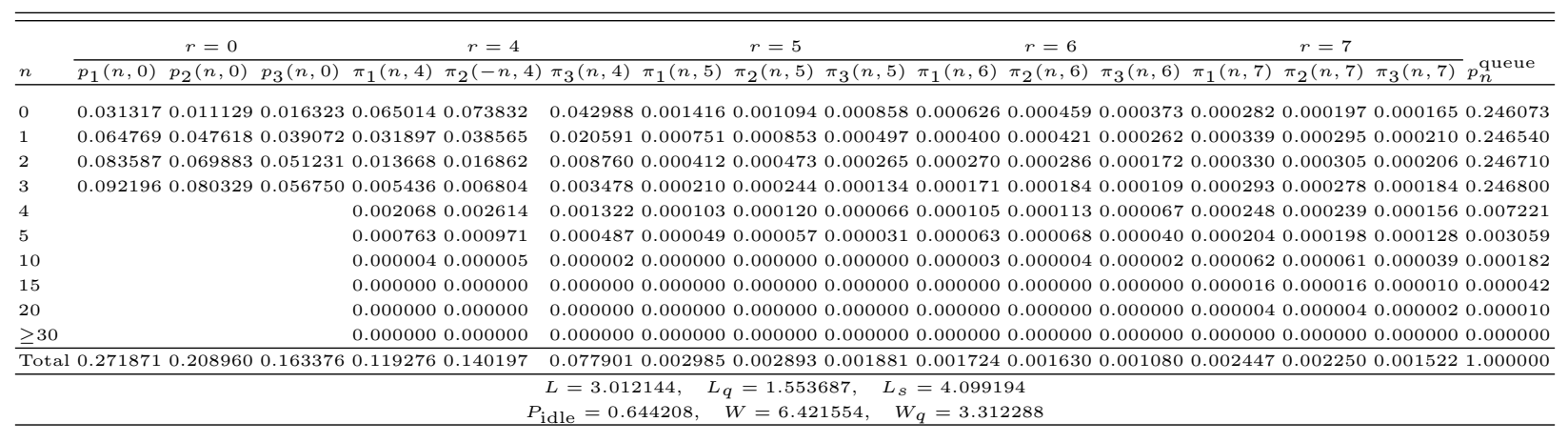

TABLE 6. Values of $\rho$ for different $\theta_{r}$.

\begin{tabular}{llll}
\hline \hline$r$ & $\theta_{r}$ & $\mu_{r}{ }^{-1}=S_{r}$ & $\rho$ \\
\hline$r=5$ & 1.810000 & 0.828729 & \\
$r=6$ & 1.551428 & 0.966851 & 0.1 \\
$r=7$ & 1.357500 & 1.104972 & \\
$r=8$ & 1.206667 & 1.243094 & \\
\hline$r=5$ & 0.904167 & 1.658986 & \\
$r=6$ & 0.775000 & 1.935484 & 0.2 \\
$r=7$ & 0.678125 & 2.211982 & \\
$r=8$ & 0.602778 & 2.488479 & \\
\hline$r=5$ & 0.604167 & 2.482759 & \\
$r=6$ & 0.517857 & 2.896552 & 0.3 \\
$r=7$ & 0.453125 & 3.310345 & \\
$r=8$ & 0.402778 & 3.724138 & \\
\hline$r=5$ & 0.452500 & 3.31491 & \\
$r=6$ & 0.387857 & 3.867403 & 0.4 \\
$r=7$ & 0.339375 & 4.419889 & \\
$r=8$ & 0.301667 & 4.972376 & \\
\hline & & &
\end{tabular}

\begin{tabular}{llll}
\hline \hline$r$ & $\theta_{r}$ & $\mu_{r}{ }^{-1}=S_{r}$ & $\rho$ \\
\hline$r=5$ & 0.362000 & 4.143646 & \\
$r=6$ & 0.310286 & 4.834254 & 0.5 \\
$r=7$ & 0.271500 & 5.524862 & \\
$r=8$ & 0.241333 & 6.215470 & \\
\hline$r=5$ & 0.301833 & 4.969630 & \\
$r=6$ & 0.258714 & 5.797902 & 0.6 \\
$r=7$ & 0.226375 & 6.626173 & \\
$r=8$ & 0.201222 & 7.454444 & \\
\hline$r=5$ & 0.258667 & 5.798969 & \\
$r=6$ & 0.221714 & 6.765464 & 0.7 \\
$r=7$ & 0.194000 & 7.731959 & \\
$r=8$ & 0.172444 & 8.698454 & \\
\hline$r=5$ & 0.226167 & 6.632277 & \\
$r=6$ & 0.193857 & 7.737656 & 0.8 \\
$r=7$ & 0.169625 & 8.843036 & \\
$r=8$ & 0.150778 & 9.948416 & \\
\hline
\end{tabular}

correlation, negative correlation and independent case (zero correlation). However, this quantity increases as $\rho$ increases. At this point, for any particular value of $\rho$, the filling degree is significantly higher for positive correlation as compared to the independent case and negative correlation.

A similar behavior can be observed in Figure 3 and 4 where the effect of correlation is studied on the average queue length and the average waiting time in the system respectively. In Figure 3 a major impact of correlation in the arrival process can be observed only when $\rho$ exceeds certain value (here $\rho>0.4$ ). However, in Figure 4 the effect can be seen even for lower values of $\rho$. These observations suggest that ignoring the correlation in the arrival process may not enable one to determine the performance measures precisely which may have a negative impact on the efficiency of the system, more specifically, when the traffic intensity is higher.

\section{Conclusion}

In this paper, we have addressed a much complicated yet significant, infinite-buffer discrete-time batch service queue with the assumption of correlated arrival process, i.e., discrete-time Markovian arrival process, with 


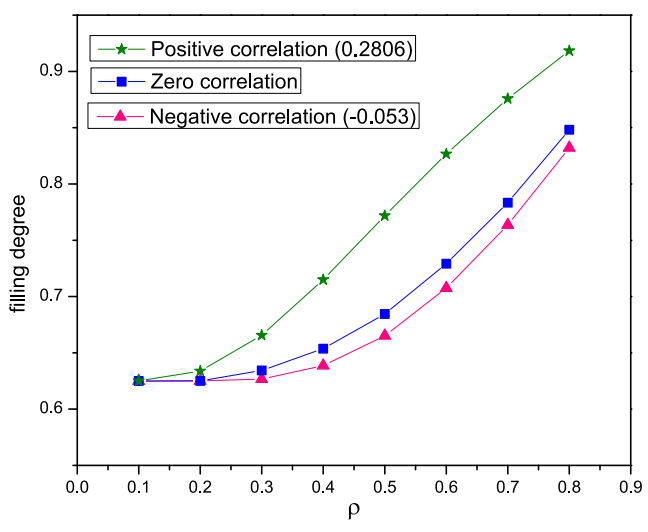

FiguRE 2. Filling degree versus $\rho$ for different correlation.

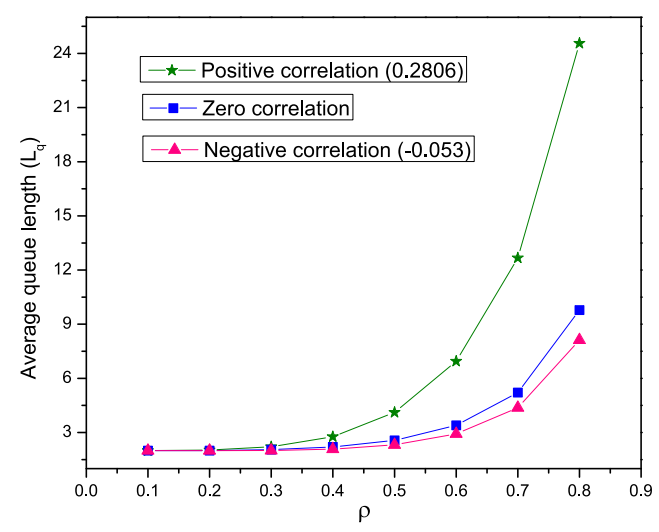

Figure 3. Average queue length $\left(L_{q}\right)$ versus $\rho$ for different correlation.

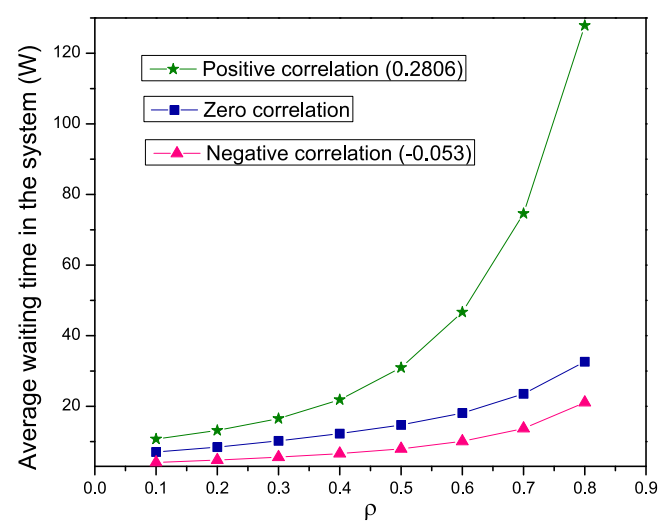

Figure 4. Average waiting time in the system $(W)$ versus $\rho$ for different correlation. 
general batch size dependent service time distribution. We have used the supplementary variable technique for the mathematical modeling and the pgf approach to obtain the probability vector generating function of the joint distribution of the queue and server content at the departure epoch. The required distribution is then extracted from the completely known generating function using the roots method, and its relation has been established with the distribution at various epochs such as arbitrary, pre-arrival and outside observer's epochs. We have discussed some significant characteristics along with some special cases of the model. The computing process is explained thoroughly and some numerical results are also presented.

Acknowledgements. The authors would like to thank the anonymous referees for their valuable comments and suggestions which led to the improvement and presentation of the paper. The research of the fifth author was supported partially by NSERC under research grant number RGPIN-2014-06604.

\section{REFERENCES}

[1] L. Abolnikov and A. Dukhovny, Optimization in HIV screening problems. Int. J. Stochastic Anal. 16 (2003) $361-374$.

[2] A.S. Alfa, Queueing Theory for Telecommunications: Discrete-Time Modelling of a Single Node System. Springer Science \& Business Media (2010).

[3] A.S. Alfa, Applied Discrete-Time Queues. Springer (2016).

[4] A. Banerjee, U.C. Gupta and V. Goswami, Analysis of finite-buffer discrete-time batch-service queue with batch-size-dependent service. Comput. Ind. Eng. 75 (2014) 121-128.

[5] S.K. Bar-Lev, M. Parlar, D. Perry, W. Stadje and F.A. Van der Duyn Schouten, Applications of bulk queues to group testing models with incomplete identification. Eur. J. Oper. Res. 183 (2007) 226-237.

[6] S.K. Bar-Lev, W. Stadje and F.A. van der Duyn Schouten, Optimal group testing with processing times and incomplete identification. Method. Comput. Appl. Probab. 6 (2004) 55-72.

[7] H. Bruneel and B.G. Kim, Discrete-Time Models for Communication Systems Including ATM. Kluwer Acadmic, Boston (1993).

[8] S.R. Chakravarthy, The batch markovian arrival process: a review and future work. In: Advances in Probability Theory and Stochastic Processes. edited by A. Krishnamoorthy et al. Notable Publications Inc., NJ (2001) 21-39.

[9] S.R. Chakravarthy, Markovian arrival processes. In: Wiley Encyclopedia of Operations Research and Management Science (2010).

[10] M.L. Chaudhry and U.C. Gupta, Analysis of a finite-buffer bulk-service queue with discrete-Markovian arrival process: D-MAP $/ G^{a, b} / 1 / N$. Nav. Res. Logistics (NRL) $50(2003) 345-363$.

[11] M.L. Chaudhry and U.C. Gupta, Queue length distributions at various epochs in discrete-time D-MAP $/ G / 1 / N$ queue and their numerical evaluations. Int. J. Inf. Manage. Sci. 14 (2003) 67-84.

[12] M.L. Chaudhry and J.G.C. Templeton, First Course in Bulk Queues. John Wiley and Sons (1983).

[13] M.L. Chaudhry, G. Singh and U.C. Gupta, A simple and complete computational analysis of MAP/R/1 queue using roots. Method. Comput. Appl. Probab. 15 (2013) 563-582.

[14] D. Claeys, B. Steyaert, J. Walraevens, K. Laevens and H. Bruneel, Analysis of a versatile batch-service queueing model with correlation in the arrival process. Perform. Eval. 70 (2013) 300-316.

[15] D. Claeys, B. Steyaert, J. Walraevens, K.D. Laevens and H. Bruneel, Tail probabilities of the delay in a batch-service queueing model with batch-size dependent service times and a timer mechanism. Comput. Oper. Res. 40 (2013) 1497-1505.

[16] H.R. Gail, S.L. Hantler, M. Sidi and B.A. Taylor, Linear independence of root equations for $M / G / 1$ type Markov chains. Queueing Syst. 20 (1995) 321-339.

[17] U.C. Gupta, S.K. Samanta and V. Goswami, Analysis of a discrete-time queue with load dependent service under discrete-time Markovian arrival process. J. Korean Stat. Soc. 43 (2014) 545-557.

[18] U.C. Gupta, G. Singh and M.L. Chaudhry, An alternative method for computing system-length distributions of $B M A P / R / 1$ and $B M A P / D / 1$ queues using roots. Perform. Eval. 95 (2016) 60-79.

[19] J.J. Hunter, Mathematical techniques of applied probability. In: Vol. 2 of Discrete Time Models: Techniques and Applications. Academic Press, New York (1983).

[20] J. Medhi, Recent Developments in Bulk Queueing Models. Wiley Eastern Limited (1984).

[21] S. Pradhan and U.C. Gupta, Modeling and analysis of an infinite-buffer batch-arrival queue with batch-size-dependent service: $M^{X} / G_{n}^{(a, b)} / 1$. Perform. Eval. 108 (2017) 16-31.

[22] S. Pradhan and U.C. Gupta, Analysis of an infinite-buffer batch-size-dependent service queue with Markovian arrival process. Ann. Oper. Res. 277 (2019) 161-196.

[23] S. Pradhan and U.C. Gupta, Stationary queue and server content distribution of a batch-size-dependent service queue with batch Markovian arrival process: $B M A P / G_{n}^{(a, b)} / 1$. To appear in: Commun. Stat. Theory Methods DOI: 10.1080/03610926.2020.1813304 (2020) 1-28.

[24] S.K. Samanta, Waiting-time analysis of $D-B M A P / G / 1$ queueing system. Ann. Oper. Res. 284 (2020) $401-413$. 
[25] H. Takagi, Queuing analysis: A Foundation of Performance Evaluation. Discrete time systems. North-Holland, Amsterdam 3 (1993).

[26] M.E. Woodward, Communication and Computer Networks: Modelling with Discrete-Time Queues. Wiley-IEEE Computer Society $\operatorname{Pr}$ (1994).

[27] M. Yu and A.S. Alfa, Algorithm for computing the queue length distribution at various time epochs in $D M A P / G^{(1, a, b)} / 1 / N$ queue with batch-size-dependent service time. Eur. J. Oper. Res. 244 (2015) 227-239. 